\title{
Inclusions in Laser Materials
}

\author{
Herbert S. Bennett \\ Institute for Materials Research, National Bureau of Standards, Washington, D.C. 20234
}

(February 16, 1971)

\begin{abstract}
One of the severe problems encountered in high-power-solid-state laser systems is the thermal damage to laser rods and optical elements. One such type of damage is thought to arise from metallic or dielectric inclusions; i.e., impurities with physical and optical properties which differ substantially from those of the host material. Such inclusions may absorb an appreciable amount of the incident radiation and thereby may undergo thermal expansion. This produces major stresses within the host material. Estimating such thermal properties requires the consideration of solutions to the heat diffusion equation and to the thermal stress equations with appropriate boundary conditions. The optical path length change for a probing light ray passing near the inclusion, the radial and tangential stress components, and the changes of the refractive index for radially polarized and tangentially polarized light due to the thermal stress field are computed. The dependence of the maximum value of the tensile stress upon the size of the inclusion and upon the physical properties of the host is examined. The feasibility of using optical techniques to detect metallic and dielectric inclusions in laser materials before they cause damage also is studied. The computations suggest that the use of laser pulse widths of the order of microseconds or longer may be more promising for the detection of small incipient absorbing centers than the use of nanosecond pulse widths.
\end{abstract}

Key words: Antimony; heat conduction; laser materials; Nd-doped glass; platinum; stress components; and thermoelastic theory.

\section{Introduction}

One of the severe problems encountered in highpower-solid-state laser systems is the internal damage of the laser rod and its optical elements. One type of such damage is the formation of interior cracks which run more or less normal to the direction of the laser beam [1]. ${ }^{1}$ This internal cracking is thought to arise from solid metallic or dielectric inclusions; i.e., impurities with physical and optical properties which differ substantially from those of the host material. There are a few documentary cases for which the laser glass around inclusions had melted and other cases for which metal was found near the center of failure cracks $[2,3]$. The metal has been usually platinum or antimony. The present theoretical study estimates the thermal stresses which occur when a solid inclusion is heated by the laser beam and examines the feasibility of using optical techniques to detect such inclusions before they cause damage. Detecting an inclusion before it causes damage eases the burden of the chemical analysis near the incipient damage area.

E. Bliss [1] has outlined the general mechanism for internal cracking. A solid inclusion with a high absorption coefficient is heated relative to the host material. The resulting thermal expansion of the inclusion may stress the surrounding host material suffi-

' Figures in brackets indicate the literature references at the end of this paper. ciently for damage to occur. In fact, the theoretical model presented in this paper predicts that the heating may produce stresses larger than the theoretical breaking stress of the glass.

Most experimental data on inclusion damage has been obtained from laser glasses which have hot platinum near the molten glass, which are processed in platinum or ceramic crucibles, and which contain neodymium as the lasing ion. The metallic inclusions in laser glasses can originate from many sources and can include most metals. However, considering the thermal properties of most metals that could occur as metallic inclusions in laser glass, one finds that they are similar in their capability for causing failure. The noble metals, in particular platinum or platinum alloys, are the metals that have been identified with any reasonable frequency in damaged laser glasses [4]. The nonmetallic inclusions may be divided into crystalline types and noncrystalline types, occluded or exsolved gas bubbles, and glassy regions. Nonmetallic inclusions can produce several weakening effects in the laser host. The most apparent of these are the lens effect, strains in flaws, electrostriction and absorption. Few definitive statements can be made about these effects and the importance of each is evaluated on a probability basis [5]. However, most researchers expect that those metallic and dielectric inclusions with high optical absorption at the lasing wavelength are most likely responsible for failure of laser glasses. The numerical results of this paper 
will treat the cases of platinum, antimony, and $\mathrm{Al}_{2} \mathrm{O}_{3}$ inclusions as representing the thermal, elastic, and optical properties of several possible inclusions in laser glass hosts. The theory will be valid, however, for other hosts which exhibit isotropic elastic properties and for other absorbing centers.

The several properties of the center-host system which determine the probability for internal cracking to occur may be divided into four groups. The first group consists of the bulk properties of both the inclusion and host and includes the respective thermal conductivities, heat capacities, thermal expansion coefficients, elastic properties, absorption coefficients for the incident radiation, and equations of state for the liquid and gas phases. The second group contains geometric properties of the inclusion which also influence its ability to cause fracture, such as the size, shape, and orientation to the incident radiation. The distribution and nature of initial microcracks and of optical imperfections form the third group of properties which determine the resistance of the host to internal cracking. Finally, the fourth group of properties describes the absorbing center-host interface; namely, the absorptance, emissivity, and initial thermal contact between the absorbing center and the host. The elastic properties, the thermal properties, and the optical properties of $\mathrm{Pt}, \mathrm{Sb}, \mathrm{Al}_{2} \mathrm{O}_{3}$, and two representative neodymium doped laser glasses are cited in tables $1,2,3,4$, and 5 .

\section{TABLE 1}

Values of the elastic parameters. The quantities $E, G, \nu$, and $\chi$ are respectively Young's modulus, shear modulus, Poisson ratio, and isothermal compressibility.

\begin{tabular}{l|c|c|c|c}
\hline \hline & $\begin{array}{c}E \\
\left(\mathrm{~N} / \mathrm{m}^{2}\right) \times 10^{10}\end{array}$ & $\begin{array}{c}G \\
\left(\mathrm{~N} / \mathrm{m}^{2}\right) \times 10^{10}\end{array}$ & $\nu$ & $\begin{array}{c}\chi \\
\left(\mathrm{m}^{2} / \mathrm{N}\right) \times 10^{-10}\end{array}$ \\
\hline $\mathrm{Pt}$ & 14.7 & 5.28 & 0.39 & 0.0449 \\
$\mathrm{Sb}$ & 7.78 & 3.25 & .20 & .234 \\
$\mathrm{Al}_{2} \mathrm{O}_{3}(\mathrm{a})$ & 41.4 & 16.2 & .275 & .0326 \\
$\mathrm{Glass}_{\text {G }}$ & 6.74 & 2.79 & .218 & .249 \\
Glass(U) & 6.74 & 2.79 & .218 & .249 \\
\hline
\end{tabular}

TABLE 2

Values of the thermal properties. The quantities $\rho, C, K$, and $\alpha$ are respectively the density, the specific heat at constant volume, the thermal conductivity, and the linear thermal expansion coefficient.

\begin{tabular}{l|c|c|c|c}
\hline \hline & $\begin{array}{c}\rho \\
\left(\mathrm{g} / \mathrm{cm}^{3}\right)\end{array}$ & $\begin{array}{c}C \\
\left(\mathrm{~J} / \mathrm{g}{ }^{\circ} \mathrm{C}\right)\end{array}$ & $\begin{array}{c}K \\
\left(\mathrm{~W} / \mathrm{cm}^{\circ} \mathrm{C}\right)\end{array}$ & $\begin{array}{c}\alpha \\
\left(1 /{ }^{\circ} \mathrm{C}\right) \times 10^{-6}\end{array}$ \\
\hline $\mathrm{Pt}(\mathrm{B})$ & 21.73 & 0.17 & 0.67 & 8.6 \\
$\mathrm{Pt}(\mathrm{U})$ & 21.73 & .13 & .67 & 9.0 \\
$\mathrm{Sb}$ & 6.62 & .21 & .18 & 11.1 \\
$\mathrm{Al}_{2} \mathrm{O}_{3}$ (a) & 4.0 & .84 & .29 & 11.0 \\
$\mathrm{Glass} \mathrm{B})$ & 2.6 & .63 & .0084 & 9.0 \\
Glass(U) & 3.0 & 1.3 & .013 & 9.0 \\
\hline
\end{tabular}

TABLE 3

Values of parameters pertaining to neglecting the coupling and inertia terms of eqs (1) and (2). The quantities $v, a^{2},\left(\delta / T_{0}\right)$, and $t_{r}$ are respectively the speed of elastic waves, diffusivity, coupling in units of the ambient temperature $T_{0}$, and the relaxation time for the deflnition of a local temperature to be meaningful.

\begin{tabular}{l|c|c|c|c}
\hline \hline & $\begin{array}{c}v \\
(\mathrm{~cm} / \mathrm{s}) \times 10^{5}\end{array}$ & $\begin{array}{c}a^{2} \\
\left(\mathrm{~cm}^{2} / \mathrm{s}\right)\end{array}$ & $\begin{array}{c}\left(\delta / T_{0}\right) \\
\left(1 /{ }^{\circ} \mathrm{C}\right) \times 10^{-5}\end{array}$ & $\begin{array}{c}t_{r} \\
(\mathrm{~s}) \times 10^{-13}\end{array}$ \\
\hline $\mathrm{Pt}$ & 3.67 & 0.238 & 4.39 & 2.0 \\
$\mathrm{Sb}$ & 3.61 & .133 & 1.67 & 2.3 \\
$\mathrm{Al}_{2} \mathrm{O}_{3}(\mathrm{a})$ & 11.44 & .0875 & 4.83 & $\sim 10.0$ \\
$\mathrm{Glass}(\mathrm{B})$ & 5.43 & .005 & 1.11 & $\sim 10.0$ \\
Glass(U) & 5.06 & .003 & 0.417 & $\sim 10.0$ \\
\hline
\end{tabular}

TABLE 4

Optical properties for $r_{0}>\lambda \approx 1.06 \mu$. The quantities $A\left(\lambda, T_{c}\right)$, $n, n^{\prime}$, and $\gamma^{-1}$ are respectively the absorptance, the real part of the refractive index, the imaginary part of the refractive index, and the inverse of the absorption coefficient.

\begin{tabular}{l|c|c|c|c}
\hline \hline & $A\left(\lambda, T_{0}\right)$ & $n$ & $n^{\prime}$ & $\begin{array}{c}\gamma^{-1} \\
(\mathrm{~cm}) \times 10^{-6}\end{array}$ \\
\hline $\mathrm{Pt}$ & 0.30 & 1.14 & 3.25 & 2.4 \\
$\mathrm{Sb}$ & .35 & 3.4 & 4.1 & 1.9 \\
$\mathrm{Al}_{2} \mathrm{O}_{3}(\mathrm{a})$ & .60 & 1.67 & $\sim 0.0$ & $($ see text) \\
$\mathrm{Glass}_{\text {G }}$ & $\ldots \ldots \ldots \ldots \ldots . .$. & 1.52 & $\sim .0$ & $\sim 4 \times 10^{8}$ \\
Glass(U) & $\ldots \ldots \ldots \ldots \ldots .$. & 1.52 & $\sim .0$ & $\sim 4 \times 10^{8}$ \\
\hline
\end{tabular}

TABLE 5

Values for the photoelastic coefficients, $p_{11}$ and $p_{12}$, the stress-optic coefficients, $B_{\|}$and $B_{4}$, and the change of index of refraction with respect to temperature, $(d n / d T)$.

\begin{tabular}{l|r|r|c|c|c}
\hline \hline & $p_{11}$ & $p_{12}$ & $\begin{array}{r}B_{\|} \\
\left(\mathrm{m}^{2} / \mathrm{N}\right) \\
\times 10^{-1.5}\end{array}$ & $\begin{array}{c}B_{\perp} \\
\left(\mathrm{m}^{2} / \mathrm{N}\right) \\
\times 10^{-1.5}\end{array}$ & $\begin{array}{c}(d n / d T) \\
\left(1 /{ }^{\circ} \mathrm{C}\right) \\
\times 10^{-6}\end{array}$ \\
\hline Glass(B) & 0.134 & 0.225 & 5.33 & 21.8 & 4.0 \\
Glass(U) & .134 & .225 & 5.33 & 21.8 & 4.0 \\
\hline
\end{tabular}

The model formulated in this paper contains many physical assumptions which are necessary to render the problem solvable. The major assumptions are summarized here and are discussed in greater detail in the following sections.

(a) The inclusion is a sphere of radius $r_{0}$ and is always in good thermal contact with the host. The number of inclusions per unit volume is assumed to be sufficiently small so that they do not interact with one another. The effects of shape and orientation to the incident radiation also are neglected in the model.

(b) The host material is isotropic, continuous, and of infinite extent. It also is initially at an ambient temperature $T_{0}$ and free from all stresses and strains. Because the energy content of the incident radiation is finite, the latter statement requires the temperature to be $T_{0}$ at infinity and all stresses and strains to vanish at infinity. The distribution and nature of microcracks and optical imperfections are not treated in the model. 
(c) The linear-thermal-elastic equations are assumed to give a reasonable description of the processes which ultimately may lead to catastrophic damage. These are coupled equations relating the temperature and the displacement vector from which the stresses and strains are computed. They are valid only when a local temperature exists and when distances are larger than atomic dimensions $\left(\sim 10^{-8} \mathrm{~cm}\right)$. A relaxation time $t_{r}$ for the definition of a local temperature $T$ is approximately the reciprocal of a characteristic vibration frequency of the material. These relaxation times $t_{r}$ for $\mathrm{Pt}, \mathrm{Sb}, \mathrm{Al}_{2} \mathrm{O}_{3}$, and the laser glasses are about $10^{-13} \mathrm{~s}$ to $10^{-12} \mathrm{~s}$. Hence, the equations are meaningful only when times $t$ are much larger than $10^{-12} \mathrm{~s}$.

(d) It is assumed that the radiation of heat by the center-host interface and by the heated glass close to the absorbing center may be neglected in the thermalelastic equations. A black body at $600{ }^{\circ} \mathrm{C}$ produces an energy flux of $0.735\left(\mathrm{~J} / \mathrm{cm}^{2} \mathrm{~s}\right)$. The laser beams for the examples studied in this paper contain energy fluxes at least $10^{4}$ times greater than $0.735\left(\mathrm{~J} / \mathrm{cm}^{2} \mathrm{~s}\right)$. The temperature gradients which occur from the time of cessation of the laser pulse to time of maximum optical path length change are always sufficiently large so that the energy flux due to thermal conduction is greater than the energy flux due to radiation. The present calculations show that the energy flux due to thermal conduction becomes small for times greater than a second and that the radiation effect should be included in the thermal-elastic equations whenever $t>1 \mathrm{~s}$. Because the temperature is close to the ambient temperature whenever the time is greater than a second, the long time behavior is not in the region of practical interest for detecting the incipient damage centers before they cause damage. Hence, it is assumed that all times are less than a second.

(e) The linear-thermal-elastic equations contain a coupling term and an inertia term. The coupling term and the inertia term may be neglected whenever the three characteristic times which occur in the absorbing center-host system satisfy a set of inequalities. These times are the following. The pulse width $\tau$ of the incident radiation determines in part the rapidity of heat generation. The characteristic relaxation time for temperature equilibration (thermal diffusion) is $t_{T}$, which is of order $\left(r^{2} / a^{2}\right)$, where $r$ is the radial distance and $a^{2}$ is the thermal diffusivity. The characteristic mechanical time required for the production of stress waves is $t_{M}$ which is of order $(r / v)$, where $v$ is the speed of propagation of elastic waves. The relationships among the coupling term, the inertia term, the time history of the displacement vector, and the time history of the temperature are complex. The easiest way in which to study these relationships is to solve exactly the linear-thermal-elastic equations for a simple one dimensional problem. Boley and Weiner have solved such a problem [6]. They demonstrate that when $\tau \gg t_{M}$ and $t_{T} \gg t_{M}$, then the coupling and inertia terms are small compared to the other terms in the equations. The data cited in table 3 for the inclusions and hosts discussed here reduce the above inequalities to inequalities containing the pulse width $\tau$ and radial distance $r$. The data show a maximum value for $t_{M} \sim 2.8 \times 10^{-6}(\mathrm{~s} / \mathrm{cm}) r$ and a minimum value for $t_{T} \sim 4.2\left(\mathrm{~s} / \mathrm{cm}^{2}\right) r^{2}$. Hence, these data lead to the inequalities containing $\tau$ and $r$; namely $\tau \gg 2.8 \times 10^{-6}$ $(\mathrm{s} / \mathrm{cm}) r$ and $r \gg 6.7 \times 10^{-7} \mathrm{~cm}$. It is assumed that these inequalities are satisfied and thereby that the coupling and inertia terms may be neglected. The results to be discussed in this paper are based upon a linear theory and upon a model whose elastic, thermal, and optical properties are constants. The values for these properties, cited in tables 1 through 5, are valid for temperatures near $300 \mathrm{~K}$ and for small strains. This treatment is not expected to be correct near the onset of damage. The problem is certainly a nonlinear one near the region of catastrophic damage. Whenever any of the assumptions become invalid, then the results should be viewed as suggesting trends in the behavior of the system. The inclusions which occur in laser glasses are most likely not the spheres for which the model has been formulated. They could be irregularly shaped globules. Some $\mathrm{Pt}$ inclusions occur as hexagonal platelets. Even though the model does not take into account such geometrical aspects, one hopes that it does give a reasonable description of the actual system. One also hopes that the manner in which it suggests one should proceed to increase the damage threshold, though probably not quantitatively correct, is qualitatively correct.

In section 2 , the linear-uncoupled-quasi-static thermal elastic equations are applied to absorbing inclusions in laser materials. The model for studying spherical inclusions is then formulated in section 3 within the context of the quasi-static thermal elastic theory. The heat diffusion equation with time dependent boundary conditions for the temperature as a function of time $t$ and of radial distance $r$ is solved by Laplace transforms. Expressions for the optical path length change for a probing beam of radiation passing near the inclusion, the radial and tangential components of the stress tensor, and the stress induced changes of the refractive index for radially and tangentially polarized light are developed in section 4. The numerical results and conclusions are presented in section 5 . Section 5 also contains an estimate for the lens effect of the heated region surrounding the absorbing center. Finally, those optical properties which determine the absorptance and emissivity of the inclusion are discussed in the appendix.

\section{Thermal Elastic Theory}

Consider an absorbing sphere of radius $r_{0}$ imbedded in an initially isotropic continuous host of infinite extent. The thermal, elastic, and mechanical properties which enter linear thermal elastic studies are: the density $\rho\left(\mathrm{g} / \mathrm{cm}^{3}\right)$, the specific heat $C$ at constant volume $\left(\mathrm{J} / \mathrm{g}{ }^{\circ} \mathrm{C}\right)$, the thermal conductivity $K\left(\mathrm{~W} / \mathrm{cm}{ }^{\circ} \mathrm{C}\right)$, the linear coefficient of thermal expansion $\alpha\left(1 /{ }^{\circ} \mathrm{C}\right)$, and the isothermal Lamé elastic constants, $\lambda$ and $\mu$. The Young's modulus $E\left(\mathrm{~N} / \mathrm{m}^{2}\right)$, the shear modulus $G\left(\mathrm{~N} / \mathrm{m}^{2}\right)$, the compressibility $\chi\left(\mathrm{m}^{2} / \mathrm{N}\right)$, and the Poisson ratio $\nu$ 
are related to the Lamé constants $\lambda$ and $\mu$ as follows,

$$
\begin{gathered}
E=(3 \lambda+2 \mu) \mu /(\lambda+\mu), \quad G=E /\{2(1+\nu)\}, \\
\chi=k^{-1}=3 /(3 \lambda+2 \mu), \quad \text { and } \quad \nu=\lambda /\{2(\lambda+\nu)\},
\end{gathered}
$$

where the bulk modulus is $k\left(\mathrm{~N} / \mathrm{m}^{2}\right)$. The derived quantities of thermal diffusivity $a^{2}=(K / \rho C)\left(\mathrm{cm}^{2} / s\right)$, the volume specific heat $\rho C\left(\mathrm{~J} / \mathrm{cm}^{3}{ }^{\circ} \mathrm{C}\right)$, and the speed of propagation of dilatational waves in an elastic medium $v=\{(\lambda+2 \mu) / \rho\}^{1 / 2}$ also enter the linear thermal elastic theory. The subscript $c$ on any of these thermal properties will refer to the absorbing center and the subscript $h$ will refer to the host.

The basic theory which describes the behavior of an absorbing sphere imbedded in a continuous medium and subjected to a laser beam combines the theories of elasticity and of heat conduction under transient conditions and is a boundary value problem of considerable mathematical difficulty. Fortunately, simplifying assumptions without significant error are possible.

The problem is that of one absorbing spherical inclusion imbedded in an infinite medium initially at a uniform temperature $T_{0}$ and subjected to a prescribed rate of internal energy absorption per unit volume $Q_{v}(\mathbf{r}, t)\left(\mathrm{W} / \mathrm{cm}^{3}\right)$. The quantity $Q_{v}$ is related to the intensity of the local radiation $I$ by an absorption equation; that is,

$$
Q_{v}(\mathbf{r}, t)=\gamma I(\mathbf{r}, t),
$$

where the linear absorption coefficient is $\gamma$ in units of inverse centimeters. This $\gamma$ should include also the effects of nonlinear absorption processes which become important at high intensities. The local intensity for radiation propagating in the plus $z$ direction at $z$ is related to the local intensity at $z_{0}$ by the relation

$$
I(z)=\exp \left\{-\gamma\left(z-z_{0}\right)\right\} I\left(z_{0}\right),
$$

where $z>z_{0}$.

The linear-thermal-elastic equations relate the temperature $T(\mathbf{r}, t)$ and the displacement vector $\mathbf{u}(\mathbf{r}, t)$ as functions of position $\mathbf{r}$ and time $t$. These coupled equations are [6]

$$
\begin{aligned}
K \nabla^{2} T-\rho C \frac{\partial T}{\partial t}-(3 \lambda+2 \mu) \alpha T_{0} \frac{\partial}{\partial t}(\nabla & \cdot \mathbf{u}) \\
& +Q_{v}=0,
\end{aligned}
$$

and

$$
\begin{aligned}
\mu \nabla^{2} \mathbf{u}+(\lambda+\mu) \nabla(\nabla \cdot \mathbf{u}) & -\rho \frac{\partial^{2} \mathbf{u}}{\partial t^{2}} \\
& -(3 \lambda+2 \mu) \alpha \nabla T=0,
\end{aligned}
$$

where $T_{0}$ is the reference temperature for which the material is free of stress. The reference temperature is chosen to be $293 \mathrm{~K}$. The strain and stress components are obtained from the displacement vector u. Equations (1) and (2) are meaningful only when a local temperature exists.

A close relationship exists between neglecting the coupling term $(3 \lambda+2 \mu) \alpha T_{0}[\partial(\nabla \cdot \mathbf{u}) / \partial t]$ and neglecting the inertia term $\rho\left(\partial^{2} \mathbf{u} / \partial t^{2}\right)$ in eqs (1) and (2). This relationship is based upon the physical idea that the rate of thermal expansion may not be sufficient to produce elastic waves. Let us consider the coupled heat equation. We rewrite eq (1) in the form

$$
\begin{array}{r}
K \nabla^{2} T-\rho C \frac{\partial T}{\partial t}\left[1+\delta \frac{(\lambda+2 \mu)\{\partial(\nabla \cdot \mathbf{u}) / \partial t\}}{(3 \lambda+2 \mu)\{\alpha(\partial T / \partial t)\}}\right] \\
+Q_{v}=0,
\end{array}
$$

where the dimensionless parameter,

$$
\delta=\left\{(3 \lambda+2 \mu)^{2} \alpha^{2} T_{0} / \rho^{2} C v^{2}\right\},
$$

determines the amount of coupling. Equation $\left(1^{\prime}\right)$ shows clearly that neglecting the coupling term requires that $\delta$ must satisfy the inequality $\delta \ll 1$ and that the strain rates, $\partial(\nabla \cdot \mathbf{u}) / \partial t$, must be less than or of the same order of magnitude as temperature rates $\alpha(\partial T / \partial t)$. The latter condition implies that the time history of the displacements must follow closely that of the temperature. In fact, the authors of ref. [6] have shown that if the application of heat is not too rapid, not only may the coupling term be omitted but the inertia term may be omitted under the same circumstances.

The rapidity of heat generation at the absorbing inclusion-host interface is characterized by the laser pulse width $\tau(\mathrm{s})$. In addition to the time $\tau$, two other characteristic times exist in the system. Namely, the characteristic thermal time, $t_{T}=\left(r^{2} / a^{2}\right)$, is the relaxation time for temperature equilibrium (thermal diffusion) and the characteristic mechanical time, $t_{M}=(r / v)$, is the time required for the production of stress waves, where the quantity $r=|\mathbf{r}|$. It has been demonstrated [6] that when

$$
\tau \gg t_{M} \quad \text { and } \quad t_{T} \gg t_{M},
$$

then coupling and inertia terms are small. That is, when inequalities (3) are satisfied, then the linear-uncoupledquasistatic thermal elastic theory obtains; namely,

$$
K \nabla^{2} T-\rho C \frac{\partial T}{\partial t}+Q_{v}=0,
$$

and

$\mu \nabla^{2} \mathbf{u}+(\lambda+\mu) \nabla(\nabla \cdot \mathbf{u})-(3 \lambda+2 \mu) \alpha \nabla T=0$,

where $\nabla^{2}$ is the Laplacian operator, and $\nabla$ is the gradient operator.

Hence neglecting the coupling term one finds that the thermal stress problem becomes two distinct problems which are solved consecutively. The first problem is the solution to the boundary value problem whose field equation is given by eq (4). When we have the temperature distribution $T(\mathbf{r}, t)$, the second problem 
is the determination of the resulting stress distribution from eq (5), and the stress-strain relations,

$$
\sigma_{i j}=\delta_{i j} \lambda \epsilon_{k k}+2 \mu \epsilon_{i j}-\delta_{i j}(3 \lambda+2 \mu) \alpha T,
$$

where $\delta_{i j}=0$ when $i \neq j$ and $\delta_{i i}=1$ when $i=j$. Equation (6) is subject to the equilibrium equations,

where

$$
\nabla \sigma_{i j}+\mathbf{f}=0
$$

$$
\epsilon_{i j}=\left(\frac{1}{2}\right)\left[\left(\partial u_{i} / \partial x_{j}\right)+\left(\partial u_{j} / \partial x_{i}\right)\right]
$$

are the strain displacement relations, $\sigma_{i j}$ is the stress tensor, and $\mathbf{f}$ is the applied force per unit volume. The subscripts $i, j$, and $k$ represent the cartesian components $x, y$, and $z$.

\section{Spherical Inclusions}

The heat diffusion eq (4) describes the time and space dependence of the temperature $T(\mathbf{r}, t)$ for large distances $r$ and large times $t$;

$$
a^{2} \nabla^{2} T=(\partial T / \partial t) .
$$

Equation (7) does not contain the volume heat generation term $Q_{v}(\mathbf{r}, t)$ because the host is assumed to be very transparent to the laser energy and to absorb only a negligible amount of energy from the laser beam. In addition, because the absorption coefficient for the absorbing center $\gamma_{c}(\mathbf{P t}$ or $\mathrm{Sb})$ is very large compared to the absorption coefficient for the host $\gamma_{h}, \gamma_{c} \sim 10^{14} \gamma_{h}$, the heat absorbed by the absorbing center from the laser beam is assumed to occur entirely at the centerhost interface. This is not the case for $\mathrm{Al}_{2} \mathrm{O}_{3}$ because $\gamma_{c}\left(\mathrm{Al}_{2} \mathrm{O}_{3}\right) \sim \gamma_{h}$ (glass). If the $\mathrm{Al}_{2} \mathrm{O}_{3}$-glass interface were highly absorbing due to some mechanism (Maxwell Demon), then the assumption that heating occurs only at the interface would be valid. The nature and existence of such a mechanism for $\mathrm{Al}_{2} \mathrm{O}_{3}$ is speculative at present. Hence the author uses in this paper the name $\mathrm{Al}_{2} \mathrm{O}_{3}$ (a) to denote an inclusion whose properties correspond to those for polycrystaline $\mathrm{Al}_{2} \mathrm{O}_{3}$ with the one exception that the absorption coefficient $\gamma_{c}\left(\mathrm{Al}_{2} \mathrm{O}_{3}(\mathrm{a})\right)$ is very much larger than that for $\gamma_{c}\left(\mathrm{Al}_{2} \mathrm{O}_{3}\right) \sim \gamma_{h}$ (glass).

The diffusion equation requires a statement of the boundary conditions before a solution is uniquely defined. The temperature $\Theta$ has the form

$$
\Theta(\mathbf{r}, t)=\left\{\begin{array}{l}
T_{0}, \quad t \leqslant 0 \\
T_{0}+T(\mathbf{r}, t), \quad t>0,
\end{array}\right.
$$

for all values of $\mathbf{r}$. The function $T(\mathbf{r}, t)$ is the temperature relative to the ambient temperature $T_{0}$. The boundary conditions are time dependent for any realistic treatment of the problem. The temperature must be finite everywhere; $T(\mathbf{r}=\infty, t)=$ finite and $T(\mathbf{r}=0, t)=$ finite. The continuity of the temperature across the center-host interface is

$$
T_{c}\left(r_{0} \hat{r}, t\right)=T_{h}\left(r_{0} \hat{r}, t\right),
$$

where $\hat{r}$ is the radial unit vector. Condition (9) implies that the host is in perfect thermal contact with the inclusion for all times $t \geqslant 0$. The conservation of heat flow at the interface gives

$-K_{c} \nabla T_{c}\left(r_{0} \hat{r}, t\right) \cdot \hat{r}+H(t ; \hat{r} \cdot \hat{z})$

$$
=-K_{h} \nabla T_{h}\left(r_{0} \hat{r}, t\right) \cdot \hat{r},
$$

where $\hat{z}$ is the unit vector in the $+z$ direction.

The energy flux $H(t ; \hat{r} \cdot \hat{z})\left(\mathrm{W} / \mathrm{cm}^{2}\right)$ is the energy absorbed by the sphere from the laser beam. Boundary condition (10) describes an uneven heating of a sphere by a plane wave propagating in the positive $z$ direction. It contains angular properties which are unnecessary refinements for our purposes, particularly when the thermal conductivity of the center is much greater than that of the host. Therefore, it is reasonable to introduce one more assumption which reduces boundary condition (10) to a spherically symmetric one. Let $E_{l}$, be the energy density $\left(\mathrm{J} / \mathrm{cm}^{2}\right)$ of the laser beam and let $\tau(\mathrm{s})$ be the pulse width of the laser beam. A square wave pulse is assumed for our problem. The spherical inclusion intercepts an area $\pi r_{0}^{2}$ from the laser beam and absorbs the amount of energy per unit time $Q_{\tau}=\left[A(\lambda, T)\left(E_{L} / \tau\right)\right] \pi r_{0}^{2}$ in watts, where $A(\lambda, T)$ is the absorptance of the center. If the sphere were to absorb energy at the rate $Q_{\tau}$ over its entire area, then the equivalent energy flux $H\left(\mathrm{~W} / \mathrm{cm}^{2}\right)$ would be $H=\left(Q_{\tau} / 4 \pi r_{0}^{2}\right)$. The equivalent energy flux for the spherically symmetric case is given by the relation,

$$
H(t)=\left\{\begin{array}{lcc}
0 \quad \text { for } & t<0 & \text { and } \quad t>\tau \\
\left(Q_{\tau} / 4 \pi r_{0}^{2}\right) & \text { for } & 0 \leqslant t \leqslant \tau .
\end{array}\right.
$$

Hence, replacing the angular dependent energy flux $H(t ; \hat{r} \cdot \hat{z})$ by the spherically symmetric energy flux $H(t)$ reduces the problem to a spherically symmetric one. Boundary condition (10) now reads

$$
-K_{c} \nabla T_{c}\left(r_{0} \hat{r}, t\right) \cdot \hat{\mathbf{r}}+H(t)=-K_{h} \nabla T_{h}\left(r_{0} \hat{r}, t\right) \cdot \hat{r} .
$$

Boundary condition (11) corresponds to replacing the incident plane wave, which has an energy flux $\left(E_{L} / \tau\right)$, with an incoming spherical wave, which has an energy flux $\left(\pi r_{0}^{2} E_{L} / 4 \pi r^{2} \tau\right)$.

The author does not include the radiation of heat by the interface and by the heated glass close to the inclusion in this model. The temperature gradients which occur from the time of cessation of the laser pulse to time of maximum optical path length change are always sufficiently large so that $\left|K_{h} \nabla T_{h}\right|$ is greater than the energy flux due to radiation. Of course, the energy flux $\left|K_{h} \nabla T_{h}\right|$ becomes small for times greater than a second and the radiation effect should be in- 
cluded in both the diffusion equation and the boundary conditions whenever $t>1 \mathrm{~s}$. Because the temperature is close to the ambient temperature whenever the time is greater than a second, the long time behavior is not in the region of practical interest for detecting the incipient damage centers before they cause damage.

The solutions for the temperature $T(\mathbf{r}, t)$ are obtained by taking the Laplace transform of the diffusion eq (7), the initial condition (8), the continuity eq (9), and the time dependent boundary condition (11). The transformed equations become linear equations for the Laplace transform $U(\mathbf{r}, s)$ of the temperature $T(\mathbf{r}, t)$, where $\mathrm{s}$ is the Laplace transform variable for the time $t$. In a separate paper [7], it has been shown that

$$
U_{h}(r, s)=B_{h} \exp \left(-z_{h}\right) / z_{h},
$$

where $z_{h}=\left(r s^{1 / 2 / a_{h}}\right)$,

$$
\begin{gathered}
B_{h}=h(s)\left(z_{h 0} / s^{1 / 2}\right) \exp \left(z_{h 0}\right) \times\left[\left(K_{h} / a_{h}\right)\left(1+z_{h 0}^{-1}\right)\right. \\
\left.-\left(K_{c} / a_{c}\right)\left(z_{c 0}^{-1}-\operatorname{ctnh} z_{c 0}\right)\right]^{-1}, \\
h(s)=\left[A\left(\lambda, T_{0}\right) E_{L} / 4 \tau\right][1-\exp (-s \tau)] s^{-1}, \\
z_{h 0}=\left(r_{0} s^{1 / 2} / a_{h}\right) \quad \text { and } \quad z_{c 0}=\left(r_{0} s^{1 / 2} / a_{c}\right) .
\end{gathered}
$$

Evaluating the Bromwich contour integral to obtain the temperature $T(\mathbf{r}, t)$ from a knowledge of the Laplace transform $U(\mathbf{r}, s)$, eqs (12) and (13), would be a most ambitious task.

Two different arguments exist by which eq (13) reduces to a more tractable form. The first argument treats eq (13) in the limit that $z_{c 0} \ll 1$, then the ctnh $\left(z_{c 0}\right)$ in eq $(13)$ is approximated by

$$
\operatorname{ctnh}\left(z_{c 0}\right) \sim z_{c 0}^{-1}+\left(z_{c 0} / 3\right)+Q\left(z_{c 0}^{3}\right)+\ldots .,
$$

and eq (12) thereby is approximated by,

$U_{h \infty}(r, s)=\operatorname{Lim}_{z_{c 0} \rightarrow 0} U_{h}(r, s)=B_{h}(\infty)\left\{\exp \left(-z_{h}\right) / z_{h}\right\}$,

where

$$
\begin{aligned}
B_{h}(\infty) & =\frac{h(s) z_{h 0} \exp \left(z_{h 0}\right)}{M\left(s^{1 / 2}+b_{+}\right)\left(s^{1 / 2}+b_{-}\right)}, \\
M & =\left(\rho_{c} C_{c} r_{0} / 3\right), b_{+}=b\left[1 \mp(1-R)^{1 / 2}\right], \\
b & =\left(K_{h} / 2 M a_{h}\right), \quad \text { and } \quad R=\left(4 \rho_{c} C_{c} / 3 \rho_{h} C_{h}\right) .
\end{aligned}
$$

The second argument yields the same relations as eqs (14) and (15) but involves simplifying the boundary condition (10). Observe that because $a_{c}^{2} \gtrsim 18 a_{h}^{2}$ and $K_{c} \gtrsim 20 K_{h}$ the temperature gradients in an absorbing center such as platinum, antimony, and $\mathrm{Al}_{2} \mathrm{O}_{3}$ (a) would be much less than those for the glass host. In the limit that $K_{c} \gg K_{h}$, the boundary condition (10) becomes

$$
\begin{array}{r}
\operatorname{Lim}_{\epsilon \rightarrow 0}\left[M\left(\partial T_{c}\left(r_{0}^{-}, t\right) / \partial t\right)\right]=\operatorname{Lim}_{\epsilon \rightarrow 0} K_{h l}\left(\partial T_{h}\left(r_{0}^{+}, t\right) / \partial r\right) \\
+H(t),
\end{array}
$$

where $r_{0}^{ \pm}=r_{0} \pm \epsilon$. Hence, approximation (14) obtains either when $\left|r_{0} s^{1 / 2} / a_{c}\right| \ll 1$ or when $K_{c} \gg K_{\prime \prime}$. The latter inequality imposes no restrictions on $r_{0}$ and $s$, but the former inequality does impose such restrictions. The volume specific heat ratio $R=\left(4 \rho_{c} C_{c} / 3 \rho_{h} C_{h}\right)$ determines three regions of behavior. Namely; when $R<1$, the roots $b \pm$ are real and unequal; when $R=1$, the roots are real and equal; and when $R>1$ the roots are complex and conjugate to one another. The evaluations of the Bromwich integral,

$T_{h}(r, t)=\operatorname{Lim}_{d \rightarrow \infty} \frac{1}{2 \pi i} \int_{c-i d}^{c+i d} \exp (s t) U_{h}(r, s) d s$,

for the above three regions appear in ref. [7], and give the temperature $T(r, t)$. The quantity $c$ is chosen to be sufficiently large in order that the integral,

$$
\int_{0}^{\infty} \exp (-c t)\left|T_{h}(r, t)\right| d t
$$

exists. Including the mathematical details for the evaluation of $T_{h}(r, t)$ in the present paper would only detract from the presentation of the model, of the physics which it contains, and of its numerical results. However, the temperature gradient in the host is negative for all values of position and time and for all finite values of the volume-specific-heat ratio $R$.

\section{Theoretical Expressions}

Having thus obtained the temperature $T(r, t)$, one may compute all the other quantities mentioned in section 1. The optical path length change $\Delta L$ becomes

$$
\Delta L\left(r_{1}\right)=\int_{L}\left(d n_{h} / d T_{h}\right) T_{h}(r, t) d x,
$$

where the integration path is along a ray that has a distance of closest approach $r_{1}$ to the absorbing sphere and the variation of the refractive index of the host with temperature is $\left(d n_{h} / d T_{h}\right)$. The quantity $\left(d n_{h} / d T_{h}\right)$ is properly the rate of change of the host refractive index with respect to temperature evaluated at zero strain. When $\left(d n_{h} / d T_{h}\right)$ is independent of $r$ and $t$, eq (19) has the form,

$\Delta L\left(r_{1} ; r_{0}\right)=\left(d n_{h} / d T_{h}\right) \int_{-D}^{+D} T_{h}\left(\left(r_{1}^{2}+x^{2}\right)^{1 / 2}, t\right) d x$,

where $T_{h}\left(\left(r_{1}^{2}+D^{2}\right)^{1 / 2}, t\right) \ll T_{h}\left(r_{0}, t\right)$ and the integration path is a straight line of length $2 D$ with a distance of closest approach to the sphere $r_{1} \geqslant r_{0}$. The stress birefringence has been neglected in eq (20). The quantities which enter the stress birefringence are computed at the end of this section. 
The stress components satisfy second order differential equations which follow by inserting the temperature from eq (17) into eq (5) and by using the relation (6). The displacement vector $\mathbf{u}=u \hat{r}$ is

$$
u(r)=\frac{\alpha(1+\nu)}{(1-\nu)} \frac{1}{r^{2}} \int_{\bar{a}}^{r} T \rho^{2} d \rho+C_{1} r+\frac{C_{2}}{r^{2}},
$$

where $\bar{a}$ is any fixed radius and where $\rho$ is the radial variable of integration. Equation (21) is correct only for those cases in which $[\{\alpha(1+\nu) /(1-\nu)\}(\partial T / \partial \rho)] \neq 0$ for $\bar{a} \leqslant \rho \leqslant r$. Because the total energy $\left(Q_{\tau} \tau\right)$ deposited by the laser beam into the center-host system is finite, conservation of energy gives

$Q_{\tau} \tau=(4 \pi / 3) \rho_{c} C_{c} r_{0}^{3} T_{h}\left(r_{0}, t\right)+4 \pi \rho_{h} C_{h} \int_{r_{0}}^{\infty} T_{h}(r, t) r^{2} d r$.

Whenever $[\{\alpha(1+\nu) /(1-\nu)\}(\partial T / \partial \rho)]=0$ for $\bar{a} \leqslant \rho \leqslant r$ then the displacement vector $\mathbf{u}=u \hat{r}$ becomes

$$
U(r)=C_{1} r+\left(C_{2} / r^{2}\right) .
$$

When $[\{\alpha(1+\nu) /(1-\nu)\}(\partial T / \partial r)] \neq 0$, then the radial stress component has the form

$$
\begin{array}{r}
\sigma_{r r}(r)=-\frac{2 \alpha E}{(1-\nu) r^{3}} \int_{\bar{a}}^{r} T \rho^{2} d \rho+\frac{E C_{1}}{(1-2 \nu)} \\
-\frac{2 E_{2}}{(1+\nu) r^{3}},
\end{array}
$$

and the tangential stress components $\sigma_{\theta \theta}$ and $\varphi_{\theta \theta}$ are equal and are given by the equation,

$$
\begin{aligned}
\sigma_{\theta \theta}(r)=\frac{\alpha E}{(1-\nu) r^{3}} \int_{\bar{a}}^{r} T \rho^{2} d \rho & +\frac{E C_{1}}{(1-2 \nu)} \\
& +\frac{E C_{2}}{(1+\nu) r^{3}}-\frac{\alpha E T}{(1-\nu)}
\end{aligned}
$$

When $[\{\alpha(1+\nu) /(1-\nu)\}(\partial T / \partial r)]=0$, then the following results,

$$
\sigma_{r r}(r)=\frac{E}{(1-2 \nu)}\left(C_{1}-\alpha T\right)-\frac{2 E C_{2}}{(1+\nu) r^{3}},
$$

and $\sigma_{\theta \theta}(r)=\sigma_{\varphi \varphi}(r)$,

$$
\sigma_{\theta \theta}(r)=\frac{E}{(1-2 \nu)}\left(C_{1}-\alpha T\right)+\frac{E C_{2}}{(1+\nu) r^{3}} .
$$

The Laplace transform given by eqs (14) and (15) corresponds to the following temperature profile:

$$
T(r, t)=\left\{\begin{array}{lcc}
T_{h}\left(r_{0}, t\right) & \text { for } & 0 \leqslant r \leqslant r_{0} \\
T_{h}(r, t) & \text { for } & r>r_{0} .
\end{array}\right.
$$

This temperature profile leads to the following expressions for $u_{c}, \sigma_{r r, c}$, and $\sigma_{\theta \theta, c}=\sigma \varphi \varphi, c$ when $r<r_{0}$ :

and

$$
U_{c}(r, t)=C_{1 c} r
$$

$\sigma_{r r, c}(r, t)=\sigma_{\theta \theta, c}(r, t)=\sigma_{\varphi \varphi, c}(r, t)$

$$
=\frac{E_{c}}{\left(1-2 \nu_{c}\right)}\left\{C_{1 c}-\alpha_{c} T_{h}\left(r_{0}, t\right)\right\},
$$

where the condition $u_{c}(\infty, t)=0$ has been used to determine $C_{2 c}=0$. The corresponding expressions for the region $r \geqslant r_{0}$ are:

$$
\begin{array}{r}
U_{h}(r, t)=\frac{\alpha_{h}\left(1+\nu_{h}\right)}{\left(1-\nu_{h}\right) r^{3}} \int_{r_{0}}^{r} T_{h}(\rho, t) \rho^{2} d \rho+\frac{C_{2 h}}{r^{2}}, \\
\sigma_{r r, h}(r, t)=-\frac{2 \alpha_{h} E_{h}}{\left(1-\nu_{h}\right) r^{3}} \int_{r_{0}}^{r} T_{h}(\rho, t) \rho^{2} d \rho \\
-\frac{2 E_{h} C_{2 h}}{\left(1+\nu_{h}\right) r^{3}},
\end{array}
$$

and

$$
\begin{array}{r}
\sigma_{\theta \theta, h}(r, t)=\sigma_{\varphi \varphi, h}(r, t)=\frac{\alpha_{h} E_{h}}{\left(1-\nu_{h}\right) r^{3}} \int_{r_{0}}^{\tau} T_{h}(\rho, t) \rho^{2} d \rho \\
+\frac{E_{h} C_{2 h}}{\left(1+\nu_{h}\right) r^{3}}-\frac{\alpha_{h} E_{h} T_{h}(r, t)}{\left(1-\nu_{h}\right)},
\end{array}
$$

where the condition $u_{h}(\infty, t)=0$ has been used to determine $C_{1 h}=0$. Observe that eqs (31) and (32) predict that $\sigma_{r r, h}(\infty, t)=0$ and $\sigma_{\theta \theta, h}(\infty, t)=0$.

The coefficients $C_{1 c}$ and $C_{2 h}$ are evaluated by introducing an adhesive boundary at $\mathbf{r}=r_{0} \hat{r}$. An adhesive boundary maintains the thermal contact between the absorbing center and the host and obtains when the following conditions are valid:

(a) The hydrostatic pressure,

$P_{c}=-\frac{1}{3}\left(\sigma_{r r, c}+\sigma_{\theta \theta, c}+\sigma_{\varphi \varphi, c}\right)=-\sigma_{r r, c}$, in the center at $\mathbf{r}=r_{0} \hat{r}$ equals the negative of the radial stress component in the host at $\mathbf{r}=r_{0} \hat{r}$; that is $\sigma_{r r, c}\left(r_{0}, t\right)=\sigma_{r r, h}\left(r_{0}, t\right)$.

(b) The tangential stress components are discontinuous across the interface.

(c) The radial displacement vector $u \hat{r}$ is continuous across the interface; that is, $u_{c}\left(r_{0}, t\right)$ $=u_{h}\left(r_{0}, t\right)$.

Conditions $a$ and $c$ then yield two simultaneous equations for $C_{1 c}$ and $C_{2 / 1}$. The solutions to this set of equations are:

$$
\frac{C_{2 h l}}{r_{0}^{3}}=\frac{\alpha_{c} T_{h}\left(r_{0}, t\right)}{1+\left\{2 E_{h} \chi_{c} / 3\left(1+\nu_{h}\right)\right\}},
$$

and

$$
C_{1 c}=\left(C_{2 h} / r_{0}^{3}\right) .
$$


A physically intuitive thought experiment which leads to the same expression for $C_{2 h}$ exists. The sphere of radius $r_{0}$ is removed from the host and is heated to $T_{h}\left(r_{0}, t\right)$ at zero pressure, $P_{i}=0$. After heating, it has an initial volume,

$$
V_{i}=(4 \pi / 3) r_{0}^{3}\left[1+3 \alpha_{c} T_{h}\left(r_{0}, t\right)\right] .
$$

The heated and expanded sphere is forced back into the host at a final pressure $P_{f}=-\sigma_{r r}, h\left(r_{0}, t\right)$. It then has a final volume

where

$$
V_{f}=(4 \pi / 3) r_{0}^{3}(1+3 \epsilon),
$$

$$
\epsilon=\left(u_{h}\left(r_{0}\right) / r_{0}\right)=\left(C_{2 h} / r_{0}^{3}\right) .
$$

Combining the above relations gives

$$
\sigma_{r r, h}\left(r_{0}, t\right)=-\left\{2 E_{h} \epsilon /\left(1+\nu_{h}\right)\right\} .
$$

Finally, the isothermal compressibility $\chi_{c}$ for the center is introduced in order to compute $\epsilon$,

$$
\chi_{c}=-(1 / V)(\partial V / \partial P)_{T} .
$$

Assuming the isothermal compressibility to be a slowly varying function of pressure for $P_{f} \geqslant P \geqslant P_{i}$ yields the relations,

$$
\begin{aligned}
\chi_{c}\left(P_{f}-P_{i}\right) & =-\ln \left(V_{f} / V_{i}\right) \\
& =-\ln \left[(1+3 \epsilon) /\left(1+3 \alpha_{c} T_{h}\left(r_{0}, t\right)\right)\right] .
\end{aligned}
$$

When $3 \epsilon<1$ and $3 \alpha_{c} T_{h}\left(r_{0}, t\right)<1$, the expansions of the logarithm give the strain $\epsilon$; namely,

$$
\epsilon \sim \frac{\alpha_{c} T_{h}\left(r_{0}, t\right)}{1+\left\{2 E_{h} \chi_{c} / 3\left(1+\nu_{h}\right)\right\}} .
$$

Substituting the coefficient $C_{2 h}=r_{0}^{3} \epsilon$ and the temperature $T_{h}(r, t)$ into eqs (31) and (32) gives the radial and tangential stress components. The radial rates of expansion during heating and of contraction during cooling for the center also are computed,

$$
r_{0} \frac{d \epsilon}{d t}=\frac{r_{0} \alpha_{c}\left\{d T_{h}\left(r_{0}, t\right) / d t\right\}}{1+\left\{2 E_{h} \chi_{c} / 3\left(1+\nu_{h}\right)\right\}} .
$$

The latter rates are used to check that the linear-quasistatic-thermal elastic theory is predicting self consistent results. If the rates of expansion or contraction of the center were to approach or exceed the speed of sound in the host, then the above problem becomes a nonlinear one involving two coupled differential equations. One equation treats heat diffusion and the other treats energy transfer in the form of sound waves.

One goal of this study is to examine under what conditions the absorbing center may initiate damage in the host. When the tensile stresses produced by the heating exceed the strength of the host, then damage should occur. The theoretical breaking stress is estimated theoretically [8] to be about one tenth of the
Young's modulus, $\sigma_{t h} \sim\left(E_{h} / 10\right)$. This value does not take into account the presence of microflaws and therefore is a representative upper limit for the strength of the host. The sign convention of the foregoing formalism is that compressive stresses are negative and tensile stresses are positive. The radial stresses are according to eq (31) always compressive (negative) and hence do not initiate damage. The tangential stresses are according to eq (32) either compressive or tensile and depend upon the time, the position, and the properties of both the center and host in a most complicated manner. Whenever the tangential stresses are tensile (positive) and are sufficiently large,

$$
\sigma_{\theta \theta, h}(r, t) \gtreqless \sigma_{t h} \sim\left(E_{h} / 10\right),
$$

they may initiate damage.

The tangential stresses at the interface may be written in the form,

$$
\sigma_{\theta \theta, h}\left(r_{0}, t\right)=E_{h} T_{h}\left(r_{0}, t\right) \delta \alpha_{\mathrm{eff}},
$$

where the effective expansion coefficient is

$$
\delta \alpha_{\mathrm{eff}}=\left[\frac{\alpha_{c}}{1+\nu_{h}+\left(2 E_{h} \chi_{c} / 3\right)}-\frac{\alpha_{h}}{\left(1-\nu_{h}\right)}\right] .
$$

It is interesting to observe that even when $\alpha_{c}=\alpha_{h}$, $\sigma_{\theta \theta, h}\left(r_{0}, t\right) \neq 0$. The stress $\sigma_{\theta \theta, h}$ is usually proportional to $\left(\alpha_{c}-\alpha_{h}\right)$ for those problems in which the temperature is spatially uniform everywhere. But in the present problem, when $r \geqslant r_{0}$, the temperature is not spatially uniform (that is, when $r \geqslant r_{0}, \nabla T_{h} \neq 0$ ) and the stress $\sigma_{\theta \theta, h}$ is not necessarily proportional to $\left(\alpha_{c}-\alpha_{h}\right)$. The model by which one reasons intuitively that the tangential stress is proportional to $\left(\alpha_{c}-\alpha_{h}\right)$ is based upon eqs (23), (26), and (27) for both $\mathrm{r}<r_{0}$ and $r>r_{0}$. This is not correct when $(\partial T / \partial r)$ differs from zero for $r \geqslant r_{0}$ as it does in the present model.

Whenever $(\partial T / \partial r)=0$ for $0 \leqslant r \leqslant \infty$, a different set of boundary conditions must be used to determine the constants in eqs (23), (26), and (27). To obtain the correct boundary conditions for the infinite host, one proceeds as follows. Consider a sphere of radius $r_{0}$ inside a spherical shell of inner radius $r_{0}$ and outer radius $r_{3}$. The system is heated adiabatically from $T_{0}$ to $T_{0}+T$ so that $(\partial T / \partial r)=0$ for $0 \leqslant r \leqslant r_{3}$. The correct equations are eqs (23), (26), and (27) and the correct boundary conditions are $u_{c}(0)=0, u_{c}\left(r_{0}\right)=u_{h}\left(r_{0}\right)$, $\sigma_{r r, c}\left(r_{0}\right)=\sigma_{r r, h}\left(r_{0}\right)$, and $\sigma_{r r, h}\left(r_{3}\right)=0$. These equations and boundary conditions then predict that in the limit as $r_{3}$ approaches infinity, the tangential stress approaches the value,

$$
\sigma_{\theta \theta, h}\left(r ; r_{3}=\infty\right)=\frac{E_{h}\left(\alpha_{c}-\alpha_{h}\right) T r_{0}^{3}}{\left\{1+\nu_{h}+\left(2 E_{\iota /} \chi_{c} / 3\right)\right\} r^{3}} .
$$

Equation (36) is not correct whenever $(\partial T / \partial r)$ differs from zero. Observe that the boundary conditions leading to eq $(36)$ replace $u_{h}(\infty)=0$ with $\sigma_{r r, h}(\infty)=0$ 
and also yield $u_{h}(\infty)=\infty$ and $\sigma_{\theta \theta, h}(\infty)=0$. Compare this with the results from eqs (30), (31), and (32); namely, $u_{h}(\infty)=0, \sigma_{r r, h}(\infty)=0$, and $\sigma_{\theta \theta, r}(\infty)=0$, because $T_{h}(r, t)$ approaches zero faster than $\left(1 / r^{2}\right)$ approaches zero as $r$ approaches infinity.

The maximum tensile stress occurs, when $\left(\partial \sigma_{\theta \theta, r}(r\right.$, $t) / \partial r)$ evaluated at $r=r_{\max }$ is zero and when $\left(\partial^{2} \sigma_{\theta \theta, h}(r\right.$, $\left.t) / \partial r^{2}\right)$ evaluated at $r=r_{\max }$ is negative. These conditions do not necessarily obtain at $r=r_{\max }=r_{0}$ and at $t=\tau$. The qualitative behavior of the tangential stress depends in part upon the sign of the effective expansion coefficient $\delta \alpha_{\text {eff }}$. When $\delta \alpha_{\text {eff }}$ is positive and sufficiently large, $\delta \alpha_{\text {eff }}>10^{-6}\left(1 /{ }^{\circ} \mathrm{C}\right)$, then the maximum tensile stress does occur at $r=r_{0}$ and $t=\tau$. For fixed times $t$, the tensile stress decreases monotonically to zero as $r$ increases from $r_{0}$. When $\delta \alpha_{\text {eff }}$ is negative, the maximum tensile stress does not occur at $r=r_{0}$ and it also does not occur necessarily at $t=\tau$. Instead, the tangential stress is always compressive at $r=r_{0}$, when $\delta \alpha_{\text {eff }}<0$. Keeping time fixed one finds that as $r$ increases from $r_{0}$ the tangential stress increases algebraically from negative (compressive) values to positive (tensile) values, attains a maximum tensile stress at $r=r_{\max }>r_{0}$, and then begins to decrease algebraically. When $\delta \alpha_{\text {eff }}$ is small, $\left|\delta \alpha_{\text {eff }}\right|$ $<10^{-6}\left(1 /{ }^{\circ} \mathrm{C}\right)$, then the stress may change from tensile to compressive or from compressive to tensile more than once as $r$ increases from $r_{0}$. This behavior for small $\delta \alpha_{\text {eff }}$ is very sensitive to the sign and value of $\left(\partial \sigma_{\theta \theta, h}(r, t) / \partial r\right)$ evaluated at $r=r_{0}$. Because evaluating $T_{h}(r, t)$ and its derivatives to study analytically the maxima of $\sigma_{\theta \theta, h}(r, t)$ would be a monumental task, the numerical results from a computer have been used to obtain the above qualitative features. The effective expansion coefficient $\delta \alpha_{\text {eff }}$ is about $-5.5 \times 10^{-6}\left(1 /{ }^{\circ} \mathrm{C}\right)$ for $\mathrm{Pt}$ in Glass $(\mathrm{B})$ and it is about $-6.7 \times 10^{-6}\left(1 /{ }^{\circ} \mathrm{C}\right)$ for $\mathrm{Sb}$ in Glass $(\mathrm{B})$.

Finally, the expressions for the strain induced change of the refractive index for radially polarized light $\Delta n_{r}$ and for tangentially polarized light $\Delta n_{\theta}=\Delta n_{\varphi}$ (stress birefringence) are developed. The theory of ref. [9] which examines the stress birefringence of an isotropiccontinuous transparent medium is used. The strain induced change of the refractive index for radially polarized light is

$$
\begin{aligned}
\Delta n_{r}(r, t)=B_{\|} \sigma_{r r, h}(r, t)+B_{+}\left(\sigma_{\theta \theta, h}(r, t)\right. \\
\left.+\sigma_{\varphi \varphi, h}(r, t)\right)+\alpha_{h} T_{h}(r, t)\left(p_{11}+2 p_{12}\right),
\end{aligned}
$$

and the strain induced change of the refractive index for tangentially polarized light is

$$
\begin{aligned}
\Delta n_{\theta}(r, t)= & \Delta n_{\varphi}(r, t)=B_{\perp} \sigma_{r r, h}(r, t) \\
& +B_{\|} \sigma_{\theta \theta, h}(r, t)+ \\
& B_{\perp} \sigma_{\varphi \varphi, h}(r, t) \\
& +\alpha_{h} T_{h}(r, t)\left(p_{11}+2 p_{12}\right),
\end{aligned}
$$

where the stress optic coefficients are

$$
B_{||}=E_{h}^{-1}\left(p_{11}-2 \nu_{h} p_{12}\right),
$$

and

$$
B_{\perp}=E_{h}^{-1}\left[\left(1-\nu_{h}\right) p_{12}-\nu_{h} p_{11}\right],
$$

and where the photoelastic coefficients are $p_{11}$ and $p_{12}$; i.e.,

and

$$
\Delta n_{r}=n_{r}-n(\epsilon=0)=p_{11} \epsilon_{r r}+p_{12}\left(\epsilon_{\theta \theta}+\epsilon_{\varphi \varphi}\right),
$$

$$
\Delta n_{\theta}=n_{\theta}-n(\epsilon=0)=p_{12}\left(\epsilon_{r r}+\epsilon_{\varphi \varphi}\right)+p_{11} \epsilon_{\theta \theta} .
$$

The stress birefringence at any point $r$ with respect to the local unit vectors for spherical coordinates is $\left(\Delta n_{r}-\Delta n_{\theta}\right)$.

Computer programs to evaluate the quantities $\Delta L, \sigma_{r r}, \sigma_{\theta \theta}, \Delta n_{r}$, and $\Delta n_{\theta}$ as functions of $r$ and $t$ for platinum, antimony, and $\mathrm{Al}_{2} \mathrm{O}_{3}$ (a) spherical inclusions are developed. Two different host glasses with properties such that both $R<1$ and $\mathrm{R}>1$ are considered. The strain rate $r_{0}(d \epsilon / d t)$ and the energy flux $S_{B}\left(T_{h}^{4}(r, t)-T_{0}^{4}\right)$ radiated by the spherical surface and by the host near the inclusion also are computed. The Stefan-Boltzman constant is denoted by $S_{B}$. For the values of radial distance and time which are important to the inclusion damage problem, the effects of both quantities are negligible and hence the model is numerically consistent with its assumptions.

\section{Results and Conclusions}

The numerical results predicted by the model developed in section 3 are reported in this section. Among the many input parameters the absorptance $A(\lambda, T)$ is perhaps most sensitive to the initial thermal contact and surface conditions of the host and inclusion. To avoid questions about the value of $A(\lambda, T)$ the numerical results for the reference (normalized) case when $A(\lambda, T)=1$ are given. This presents no additional problem because the temperature $T_{h}(r, t)$, the optical path length change $\Delta L$, the stress components $\sigma_{r r}$ and $\sigma_{\theta \theta}=\sigma_{\varphi \varphi}$ and the stress birefringence $\Delta n_{r}$ and $\Delta n_{\theta}$, are all directly proportional to the absorptance.

The model of section 3 has been used to study two different questions. First, how the maximum tangentialtensile stress varies as a function of the radius of the spherical inclusion for a fixed energy density and pulse width of the laser beam. Second, if the maximum temperature of the inclusion is limited to a fixed value, $T_{h}\left(r=r_{0}, t=\tau\right)=$ constant, how the parameters of the incident laser beam should be varied to increase the probability of detecting by optical techniques a small incipient absorbing center, before it causes damage. Optical techniques to detect small inclusions become more promising the greater $\Delta L, \Delta n_{r}$, and $\Delta n_{\theta}$ are.

Table 6 for inclusions in Glass(B) and table 7 for inclusions in Glass $(\mathrm{U})$ contain the variation of the maximum tensile stress $\sigma_{\theta \theta}$ (max-tensile) as a function of $r_{0}$ for a laser beam having an energy density $20\left(\mathrm{~J} / \mathrm{cm}^{2}\right)$ and a pulse width $30 \mathrm{~ns}$. When $10^{-4} \mathrm{~cm}>r_{0}>5 \times 10^{-5}$ $\mathrm{cm}$ the maximum tensile stress exceeds the theoretical strength of the Glass(B). Also when $10^{-4} \mathrm{~cm}>r_{0}>5$ 
$\times 10^{-5} \mathrm{~cm}$ the maximum tensile stress exceeds the theoretical strength of the Glass(U). These results demonstrate that submicron-sized inclusions have the greatest probability to cause damage in laser glass hosts. The results given for $r_{0}=10^{-7} \mathrm{~cm}$ may have no physical meaning because the uncoupled thermoelastic theory becomes suspect for $r_{0} \lesssim 6.7 \times 10^{-7} \mathrm{~cm}$. Whenever the temperature $T_{h}\left(r_{0}, \tau\right)$ exceeds the strain point of the glass, the results reported in tables 6 and 7 are at best order of magnitude estimates for the tensile stress. Laser glasses have strain points around $600{ }^{\circ} \mathrm{C}$. These two tables show that very large and very small inclusions are not likely to produce damage. Only inclusions having an intermediate size of the order of tenths of microns have a high probability to produce damage. They also show that the surface temperature $T_{h}\left(r_{0}, \tau\right)$ and the maximum tensile stress become independent of the thermal and elastic properties of the inclusion when $r_{0}<10^{-6} \mathrm{~cm}$. This observation is proven in ref. [7] by analytic methods.

The high temperatures which are predicted by this model for $0.1 \mu \leqslant r_{0} \leqslant 1 \mu$ are certainly not to be taken seriously. The model does not treat the heats of fusion $H_{F}$, the heats of vaporization $H_{V}$, the changes in volume upon melting, and the equation of state for the liquidgas transition at extremely high temperatures. These equations of state for platinum, antimony, and $\mathrm{Al}_{2} \mathrm{O}_{3}$ are not known. Also some researchers argue that these effects which the present model neglects tend to cancel one another in the case of platinum for temperatures up to its boiling point and that thereby, even though the model predicts temperatures which are too large, it does give representative values for the stresses. Neglecting the possible chemical and physical changes which may occur at the center-host interface, they argue as follows. The heat of fusion $H_{F}(c)$ gives an effective temperature of fusion $T_{F}(c)=H_{F}(c) / C_{c}$ by which the temperature $T_{h}\left(r_{0}, \tau\right)$, given by the model, is too large whenever $T_{M}(c)<T_{h}\left(r_{0}, \tau\right)<T_{B}(c)$, where $T_{M}(c)$ and $T_{B}(c)$ are respectively the melting and boiling points of the center. The values for platinum are $T_{F}(\mathrm{Pt}) \sim 800{ }^{\circ} \mathrm{C}, T_{M}(\mathrm{Pt}) \sim 1770{ }^{\circ} \mathrm{C}$ and $T_{B}(\mathrm{Pt})$ $\sim 3830{ }^{\circ} \mathrm{C}$. The excess temperature $T_{F}(\mathrm{Pt})$ predicted by the present model produces an excess volume change $(\Delta V \mid V)_{\mathrm{ex}} \sim 3 \alpha_{c} T_{F}(\mathrm{Pt}) \sim+0.02$. But the volume change for the platinum upon melting is also about $+0.02[9]$. Hence, the model should give correct order of magnitude estimates for platinum up to its boiling point. Such arguments fail for antimony because its $(\Delta V / V)$ upon melting is about -0.014 .

The following mesh in the $r$ and $t$ space is used to obtain the values given in tables 6 to 9 . The expressions are evaluated for five values of the time $(3 \mathrm{~ns}, 30 \mathrm{~ns}$, $0.3 \mu \mathrm{s}, 3 \mu \mathrm{s}$, and $30 \mu \mathrm{s})$ and for twenty values of $r$. The limits on $r$ depend upon $r_{0}$. When $r_{0} \geqslant 0.1 \mu$, the limits are $r_{0} \leqslant r \leqslant 5 r_{0}$; when $r_{0}=0.01 \mu$ they are $r_{0} \leqslant r$ $\leqslant 60 r_{0}$; and when $r_{0}=0.001 \mu$, they are $r_{0} \leqslant r \leqslant 600 r_{0}$. The twenty values of $r$ are equally spaced in a logarithmic manner between the lower and upper limits.

The quantities $\Delta L\left(r, r_{0}, t\right), \sigma_{r r}(r, t), \sigma_{\theta \theta}(r, t)$, $\Delta n_{r}(r, t)$ and $\Delta n_{\theta}(r, t)$ have been evaluated for $r_{0}=0.1 \mu, 1 \mu$, and $10 \mu$, and $T_{h}\left(r_{0}, \tau\right)=600{ }^{\circ} \mathrm{C}$; for seven values of $\tau, 3 \mathrm{~ns} \leqslant \tau \leqslant 3 \mathrm{~ms}$; for ten values of $r, r_{0} \leqslant r \leqslant 0.2 \mathrm{~cm}$; and for ten values of time $3 \mathrm{~ns}$ $\leqslant t \leqslant 3 \mathrm{~s}$, where $\tau, r$, and $t$ are all equally spaced in a logarithmic manner. The presentation in this paper of all these numerical results would be overwhelming. Table 10 contains a summary of these results for the case of platinum in Glass(B). The results for antimony and $\mathrm{Al}_{2} \mathrm{O}_{3}(a)$ in Glass(B) and Glass(U) and for platinum in Glass $(\mathrm{U})$, are similar to within a factor of ten.

\section{TABLE 6}

The maximum tensile stress as a function of $r_{0}$ for fixed energy density $E_{L}=20\left(\mathrm{~J} / \mathrm{cm}^{2}\right)$ and pulse width $\tau=30 \mathrm{~ns}$. The theoretical breaking stress of glass is about $6 \times 10^{9}\left(\mathrm{~N} / \mathrm{m}^{2}\right)$. The properties of Glass $(B)$ are used in this table. The radius of the inclusion is $r_{0}$; the maximum tensile stress is $\sigma_{\theta \theta}$ (max-tensile) and occurs at position $r$ and at time $t$; the temperature at $r$ and $t$ is $T_{h}(r, t)$; the volume specific heat ratio is $R$; and the effective expansion coefficient is $\delta \alpha_{\text {eff }}$.

Inclusion: $\mathrm{Pt}(\mathrm{B}), R=2.97, \delta \alpha_{\mathrm{eff}}=-5.5 \times 10^{-6}\left(1 /{ }^{\circ} \mathrm{C}\right)$

\begin{tabular}{c|c|c|c|c}
\hline $\begin{array}{c}\log _{10}\left(r_{0}\right) \\
\left(r_{0} \text { in cm }\right)\end{array}$ & $\begin{array}{c}\log _{10}(t / 3) \\
(t \text { in s) }\end{array}$ & $\left(r / r_{0}\right)$ & $\begin{array}{c}T_{h}(r, t) \\
\left({ }^{\circ} \mathrm{C}\right)\end{array}$ & $\begin{array}{c}\sigma_{\theta \theta}(\mathrm{max}-\mathrm{tensile}) \\
\left(\mathrm{N} / \mathrm{m}^{2}\right) \times 10^{9}\end{array}$ \\
\hline & & & & \\
-2.0 & -8.0 & 1.1 & 0.0 & 0.13 \\
-3.0 & -8.0 & 1.1 & .0 & 1.3 \\
-4.0 & -8.0 & 1.3 & 910. & 7.6 \\
-4.3 & -8.0 & 1.4 & 5200. & 9.0 \\
-5.0 & -8.0 & 3.3 & 370. & 2.6 \\
-6.0 & -9.0 & 11. & 98. & 0.098 \\
-7.0 & -9.0 & 110. & 0.80 & .00097 \\
\hline
\end{tabular}

Inclusion: $\mathrm{Sb}, R=1.13, \delta \alpha_{\mathrm{eff}}=-6.7 \times 10^{-6}\left(1 /{ }^{\circ} \mathrm{C}\right)$

\begin{tabular}{c|c|c|c|c}
\hline $\begin{array}{c}\log _{10}\left(r_{0}\right) \\
\left(r_{0} \text { in } \mathrm{cm}\right)\end{array}$ & $\begin{array}{c}\log _{10}(t / 3) \\
(t \text { in s) }\end{array}$ & $\left(r / r_{0}\right)$ & $\begin{array}{c}T_{h}(r,-t) \\
\left({ }^{\circ} \mathrm{C}\right)\end{array}$ & $\begin{array}{c}\sigma_{\theta \theta}(\mathrm{max}-\text { tensile }) \\
\left(\mathrm{N} / \mathrm{m}^{2}\right) \times 10^{9}\end{array}$ \\
\hline & & & & \\
-2.0 & -8.0 & 1.1 & 0.0 & 0.27 \\
-3.0 & -8.0 & 1.1 & .0 & 2.7 \\
-4.0 & -8.0 & 1.3 & 2100. & 13. \\
-4.3 & -8.0 & 1.3 & 4300. & 13. \\
-5.0 & -9.0 & 1.8 & 1600. & 3.4 \\
-6.0 & -9.0 & 11. & 100. & 0.097 \\
-7.0 & -9.0 & 110. & 0.80 & .00097 \\
\hline
\end{tabular}

Inclusion: $\mathrm{Al}_{2} \mathrm{O}_{3}(\mathrm{a}), R=2.74, \delta \alpha_{\text {eff }}=-3.4 \times 10^{-6}\left(1 /{ }^{\circ} \mathrm{C}\right)$

\begin{tabular}{c|c|c|c|c}
\hline $\begin{array}{c}\log _{10}\left(r_{0}\right) \\
\left(r_{0} \text { in cm }\right)\end{array}$ & $\begin{array}{c}\log _{10}(t / 3) \\
(t \text { in s })\end{array}$ & $\left(r / r_{0}\right)$ & $\begin{array}{c}T_{h}(r, t) \\
\left({ }^{\circ} \mathrm{C}\right)\end{array}$ & $\begin{array}{c}\sigma_{\theta \theta}(\mathrm{max}-\text { tensile }) \\
\left(\mathrm{N} / \mathrm{m}^{2}\right) \times 10^{9}\end{array}$ \\
\hline & & & & \\
-2.0 & -8.0 & 1.1 & 0.0 & 0.19 \\
-3.0 & -8.0 & 1.1 & .0 & 1.9 \\
-4.0 & -8.0 & 1.2 & 4600. & 11. \\
-4.3 & -8.0 & 1.4 & 5600. & 13. \\
-5.0 & -9.0 & 1.5 & 3500. & 3.6 \\
-6.0 & -9.0 & 11. & 98. & 0.10 \\
-7.0 & -9.0 & 110. & 0.80 & .00096 \\
\hline
\end{tabular}


The maximum tensile stress as a function of $r_{0}$ for fixed energy density $E_{L}=20\left(\mathrm{~J} / \mathrm{cm}^{2}\right)$ and pulse width $\tau=30 \mathrm{~ns}$. The theoretical breaking stress of glass is about $6 \times 10^{9}\left(\mathrm{~N} / \mathrm{m}^{2}\right)$. The properties for Glass $(\mathrm{U})$ are used in this table. The radius of the inclusion is $r_{0}$; the maximum tensile stress is $\sigma_{\theta \theta}$ (max-tensile) and occurs at position $r$ and at time $t$; the temperature at $r$ and $t$ is $T_{h}(r, t)$; the volume specific heat ratio is $R$; and the effective expansion coefficient is $\delta \alpha_{\text {eff }}$.

Inclusion: $\operatorname{Pt}(\mathrm{U}), R=0.97, \delta \alpha_{\text {eff }}=-5.1 \times 10^{-6}\left(1 /{ }^{\circ} \mathrm{C}\right)$

\begin{tabular}{c|c|c|c|c}
\hline $\begin{array}{c}\log _{10}\left(r_{0}\right) \\
\left(\mathrm{r}_{0} \text { in } \mathrm{cm}\right)\end{array}$ & $\begin{array}{c}\log _{10}(t / 3) \\
(t \text { in } \mathrm{s})\end{array}$ & $\left(r / r_{0}\right)$ & $\begin{array}{c}T_{h}(r, t) \\
\left({ }^{\circ} \mathrm{C}\right)\end{array}$ & $\begin{array}{c}\sigma_{\theta \theta}(\mathrm{max}-\mathrm{tensile}) \\
\left(\mathrm{N} / \mathrm{m}^{2}\right) \times 10^{9}\end{array}$ \\
\hline-2.0 & -8.0 & 1.1 & 0.0 & 0.18 \\
-3.0 & -8.0 & 1.1 & .0 & 1.8 \\
-4.0 & -8.0 & 1.2 & 2800. & 10. \\
-4.3 & -8.0 & 1.4 & 2900. & 11. \\
-5.0 & -9.0 & 1.5 & 1900. & 2.8 \\
-6.0 & -9.0 & 8.6 & 87. & 0.081 \\
-7.0 & -9.0 & 80. & 1.3 & .00081 \\
\hline
\end{tabular}

Inclusion: $\mathrm{Sb}, R=0.49, \delta \alpha_{\mathrm{eff}}=-6.7 \times 10^{-6}\left(1 /{ }^{\circ} \mathrm{C}\right)$

\begin{tabular}{c|c|c|c|c}
\hline $\begin{array}{c}\log _{10}\left(r_{0}\right) \\
\left(r_{0} \text { in } \mathrm{cm}\right)\end{array}$ & $\begin{array}{c}\log _{10}(t / 3) \\
(t \text { in s })\end{array}$ & $\left(r / r_{0}\right)$ & $\begin{array}{c}T_{h}(r, t) \\
\left({ }^{\circ} \mathrm{C}\right)\end{array}$ & $\begin{array}{c}\sigma_{\theta \theta}(\max -\text { tensile }) \\
\left(\mathrm{N} / \mathrm{m}^{2}\right) \times 10^{9}\end{array}$ \\
\hline-2.0 & -8.0 & 1.1 & 0.0 & 0.27 \\
-3.0 & -8.0 & 1.1 & .0 & 2.6 \\
-4.0 & -8.0 & 1.2 & 4600. & 11. \\
-4.3 & -8.0 & 1.2 & 4300. & 11. \\
-5.0 & -9.0 & 1.7 & 1200. & 2.7 \\
-6.0 & -9.0 & 8.6 & 89. & 0.079 \\
-7.0 & -9.0 & 80. & 1.3 & .00081 \\
\hline
\end{tabular}

Inclusion: $\mathrm{Al}_{2} \mathrm{O}_{3}(\mathrm{a}), R=1.19, \delta \alpha_{\text {eff }}=-3.4 \times \mathrm{i}^{-6}\left(1 /{ }^{\circ} \mathrm{C}\right)$

\begin{tabular}{c|c|c|c|c}
\hline $\begin{array}{c}\log _{10}\left(r_{0}\right) \\
\left(r_{0} \text { in } \mathrm{cm}\right)\end{array}$ & $\begin{array}{c}\log _{10}(t / 3) \\
(t \text { in s) }\end{array}$ & $\left(r / r_{0}\right)$ & $\begin{array}{c}T_{h}(r, t) \\
\left({ }^{\circ} \mathrm{C}\right)\end{array}$ & $\begin{array}{c}\sigma_{\theta \theta}(\max -t e n s i l e) \\
\left(\mathrm{N} / \mathrm{m}^{2}\right) \times 10^{9}\end{array}$ \\
\hline-2.0 & -8.0 & 1.1 & 0.0 & 0.19 \\
-3.0 & -8.0 & 1.1 & .0 & 1.9 \\
-4.0 & -8.0 & 1.2 & 2400. & 11. \\
-4.3 & -8.0 & 1.3 & 6900. & 12. \\
-5.0 & -9.0 & 1.5 & 1700. & 3.3 \\
-6.0 & -9.0 & 8.6 & 86.2 & 0.083 \\
-7.0 & -9.0 & 80. & 1.3 & .00081 \\
\hline
\end{tabular}

Table 10 contains the optical path length change $\Delta L$ and the stress birefringence parameters $\left(\Delta n_{r}\right.$ and $\left.\Delta n_{\theta}\right)$ which occur when $\mathrm{Pt}$ is in Glass(B) for several values of pulse width $\tau$ and inclusion size $r_{0}$. The results for $\mathrm{Pt}$ in Glass $(\mathrm{U})$ are similar within a factor of about two to those predicted for Glass(B) and are not included here. Because the model assumes that the strain is proportional to stress, the energy densities $E_{L}$, of the laser pulses are limited in table 10 to values such that the temperature of the glass never exceeds the strain point of the glass. That is, the energy densities are such that $T_{h}\left(r_{0}, \tau\right)=600{ }^{\circ} \mathrm{C}$. In addition, the maximum tensile stress is always at least an order of mangitude less than the theoretical breaking point of the host $6 \times 10^{9}\left(\mathrm{~N} / \mathrm{m}^{2}\right)$. Hence, excluding the complications which microcracks might produce, one does not expect damage to occur when $T_{h}\left(r_{0}, \tau\right)$ $\leqslant 600^{\circ} \mathrm{C}$. The quantities $\langle\Delta L\rangle,\left\langle\Delta n_{r}\right\rangle$, and $\left\langle\Delta n_{\theta}\right\rangle$
Maximum tensile stress of $\mathrm{Pt}$ spheres in a fictitious host as a function of the thermal expansion coefficient of the host. All the properties of the host are the same as those for Glass(B) except that the thermal expansion coefficient of the host $\alpha_{h}$ varies from $6 \times 10^{-6}$ $\left(1 /{ }^{\circ} \mathrm{C}\right)$ to $16 \times 10^{-6}\left(1 /{ }^{\circ} \mathrm{C}\right)$. The effective expansion coefficient is $\delta \alpha_{\text {eff }}$; the maximum tensile stress at a distance $r$ and at time $t$ is $\sigma_{\theta \theta}$ (max-tensile); and the temperature of the host is $T_{h}(r, t)$. The, laser pulse width is $\tau=30 \mathrm{~ns}$ and its energy density is $E_{L}=20\left(\mathrm{~J} / \mathrm{cm}^{2}\right)$. The radius of the sphere is $r_{0}=5 \times 10^{-5} \mathrm{~cm}$. The maximum stress occurs for times close to the pulse width, $t \sim \tau$.

\begin{tabular}{c|c|c|c|c}
\hline \hline $\begin{array}{c}\alpha_{h} \\
\left(1 /{ }^{\circ} \mathrm{C}\right) \times 10^{-6}\end{array}$ & $\begin{array}{c}\delta \alpha_{\text {eff }} \\
\left(1 /{ }^{\circ} \mathrm{C}\right) \times 10^{-6}\end{array}$ & $r / r_{0}$ & $\begin{array}{c}\sigma_{\theta \theta}(\text { max-tensile }) \\
\left(\mathrm{N} / \mathrm{m}^{2}\right) \times 10^{9}\end{array}$ & $\begin{array}{c}T_{h}(r, t) \\
\left(\times 10^{3}{ }^{\circ} \mathrm{C}\right)\end{array}$ \\
\hline & & & & \\
6.0 & -1.6 & 1.4 & 9.0 & 5.2 \\
8.0 & -4.2 & 1.4 & 8.9 & 5.2 \\
10. & -6.7 & 1.4 & 8.8 & 5.2 \\
12. & -9.3 & 1.5 & 9.1 & 2.1 \\
14. & -12. & 1.5 & 9.3 & 2.1 \\
16. & -14. & 1.5 & 9.7 & 2.1 \\
\hline
\end{tabular}

TABLE 9

Maximum tensile stress of $\mathrm{Pt}$ spheres in a fictitious host as a func tion of the thermal expansion coefficient of the host. All the properties of the host are the same as those for Glass(U) except that the thermal expansion coefficient of the host $\alpha_{h}$ varies from $4 \times 10^{-6}$ $\left(1 /{ }^{\circ} \mathrm{C}\right)$ to $12 \times 10^{-6}\left(1 /{ }^{\circ} \mathrm{C}\right)$. The effective expansion coefficient is $\delta \alpha_{\text {eff }}$; the maximum tensile stress at a distance $r$ and at time $t$ is $\sigma_{\theta \theta}$ (max-tensile); and the temperature of the host is $T_{h}(r, t)$. The laser pulse width is $\tau=30 \mathrm{~ns}$ and its energy density is $E_{L}=20\left(\mathrm{~J} / \mathrm{cm}^{2}\right)$. The radius of the sphere is $r_{0}=5 \times 10^{-5} \mathrm{~cm}$. The maximum tensile stress occurs for times close to the pulse width, $t \sim \tau$.

\begin{tabular}{c|c|c|c|c}
\hline \hline $\begin{array}{c}\alpha_{h} \\
\left(1 /{ }^{\circ} \mathrm{C}\right) \times 10^{-6}\end{array}$ & $\begin{array}{c}\delta \alpha_{\text {eff }} \\
\left(1 /{ }^{\circ} \mathrm{C}\right) \times 10^{-6}\end{array}$ & $r / r_{0}$ & $\begin{array}{c}\sigma \text { (max-tensile }) \\
\left(\mathrm{N} / \mathrm{m}^{2}\right) \times 10^{9}\end{array}$ & $\begin{array}{c}T_{h}(r, t) \\
\left(\times 10^{3}{ }^{\circ} \mathrm{C}\right)\end{array}$ \\
\hline & & & & \\
4. & 1.2 & 1.3 & 12. & 7.9 \\
6. & -1.3 & 1.3 & 11. & 7.9 \\
8. & -3.9 & 1.4 & 10. & 2.9 \\
10. & -6.4 & 1.4 & 11. & 2.9 \\
12. & -9.0 & 1.4 & 11. & 2.9 \\
\hline
\end{tabular}

denote representative values of the quantities $\Delta L\left(r_{1}\right.$, $\left.r_{0}, t\right), \Delta n_{r}(r, t)$, and $\Delta n_{\theta}(r, t)$ for those regions of $r_{0} \leqslant r \leqslant r_{2}$ and $t_{1} \leqslant t \leqslant t_{2}$ in which they obtain their largest values. The meaning of these symbols is illustrated below for one case from table 10. For example, the largest optical path length changes due to a platinum sphere of radius $r_{0}=1 \mu$ heated by a laser beam having a pulse width $3 \times 10^{-8} \mathrm{~s}$ and an energy density $0.33\left(\mathrm{~J} / \mathrm{cm}^{2}\right)$ are about $1.3 \times 10^{-8} \mathrm{~cm}$ and occur for times between $3 \mu \mathrm{s}$ and $30 \mu \mathrm{s}$ after the beginning of the laser pulse and for optical paths which approach within $l \mu$ of the surface of the inclusion. Similarly, the largest values for the radial and tangentail changes of the index of refraction due to thermal birefringence are respectively $6.3 \times 10^{-4}$ and $6.0 \times 10^{-4}$ and occur for the same radial distance and time as those for the optical path length changes. 
Optical path length change and stress birefringence for Pt inclusions in Glass(B). The radius of the inclusion is $r_{0}(\mathrm{~cm})$, the pulse width is $\tau(\mathrm{s})$, the energy density is $E_{l}\left(\mathrm{~J} / \mathrm{cm}^{2}\right)$, and the optical path length change at time $t=\tau$ and for a distance of closest approach $r=r_{0}$ is $\Delta L\left(r_{0}, r_{0}, \tau\right)(\mathrm{cm})$. The quantities $\langle\Delta L\rangle(\mathrm{cm}),\left\langle\Delta n_{r}\right\rangle$, and $\left\langle\Delta n_{\theta}\right\rangle$ denote respectively representative values for the largest optical path length change, the largest index change for radially polarized light, and the largest index change for tangentially polarized light which occur for a range of distances of closest approach $r_{0}$ to $r_{2}(\mathrm{~cm})$ and for an interval of time $t_{1}$ to $t_{2}(\mathrm{~s})$ after the beginning of the laser pulse. The tangential stress at $r=r_{0}$ and $t=\tau$ is $-2.16 \times 10^{8}\left(\mathrm{~N} / \mathrm{m}^{2}\right)$ and the surface temperature $T_{h}\left(r_{0}, \tau\right)$ at $t=\tau$ is $600{ }^{\circ} \mathrm{C}$ in all cases. The radius is $r_{0}=10^{-5} \mathrm{~cm}$.

\begin{tabular}{c|c|c|c|c|c|c|c|c}
\hline \hline $\begin{array}{c}\log _{10}(\tau / 3) \\
(\tau \text { in s })\end{array}$ & $\begin{array}{c}E_{L} \\
\left(\mathrm{~J} / \mathrm{cm}^{2}\right)\end{array}$ & $\begin{array}{c}\log _{10}\left(t_{1} / 3\right) \\
\left(t_{1} \text { in s }\right)\end{array}$ & $\begin{array}{c}\log _{10}\left(t_{2} / 3\right) \\
\left(t_{2} \text { in s }\right)\end{array}$ & $\begin{array}{c}\log _{10}\left(r_{2}\right) \\
\left(r_{2} \text { in } \mathrm{cm}\right)\end{array}$ & $\begin{array}{c}\Delta L\left(r_{0}, r_{0}, \tau\right) \\
(\mathrm{cm}) \times 10^{-6}\end{array}$ & $\begin{array}{c}\langle\Delta L\rangle \\
(\mathrm{cm}) \times 10^{-6}\end{array}$ & $\begin{array}{c}\left\langle\Delta n_{r}\right\rangle \\
\times 10^{-4}\end{array}$ & $\begin{array}{c}\left\langle\Delta n_{H}\right\rangle \\
\times 10^{-4}\end{array}$ \\
\hline-3 & 6035. & -4 & -3 & -4 & 0.24 & 0.20 & 2.1 & 1.7 \\
-4 & 605. & -5 & -4 & -4 & .23 & .14 & 2.0 & 1.6 \\
-5 & 61.1 & -6 & -5 & -4.7 & .19 & .14 & 1.9 & 1.5 \\
-6 & 6.32 & -7 & -6 & -4.7 & .14 & .088 & 5.8 & 4.5 \\
-7 & 0.706 & -7 & -6 & -4.7 & .094 & .033 & 4.7 & 3.2 \\
-8 & .113 & -7 & -6 & -4.7 & .047 & .0049 & 0.022 & 0.021 \\
-9 & .0446 & -7 & -6 & -4.7 & .025 & .0019 & .0080 & .0076 \\
\hline
\end{tabular}

\section{TABLE 10B}

Optical path length change and stress birefringence for Pt inclusions in Glass(B). The notation of this table is the same as that for table $10 \mathrm{a}$. The radius is $r_{0}=10^{-4} \mathrm{~cm}$.

\begin{tabular}{c|c|c|c|c|c|c|c|c}
\hline \hline $\begin{array}{c}\log _{10}(\tau / 3) \\
(\tau \text { in s })\end{array}$ & $\begin{array}{c}E_{l} \\
\left(\mathrm{~J} / \mathrm{cm}^{2}\right)\end{array}$ & $\begin{array}{c}\log _{10}\left(t_{1} / 3\right) \\
\left(t_{1} \text { in s }\right)\end{array}$ & $\begin{array}{c}\log _{10}\left(t_{2} / 3\right) \\
\left(t_{2} \text { in s }\right)\end{array}$ & $\begin{array}{c}\log _{10}\left(r_{2}\right) \\
\left(r_{2} \text { in } \mathrm{cm}\right)\end{array}$ & $\begin{array}{c}\Delta L\left(r_{0}, r_{0}, \tau\right) \\
(\mathrm{cm}) \times 10^{-6}\end{array}$ & $\begin{array}{c}\langle\Delta L\rangle \\
(\mathrm{cm}) \times 10^{-6}\end{array}$ & $\begin{array}{c}\left\langle\Delta n_{r}\right\rangle \\
\times 10^{-4}\end{array}$ & $\begin{array}{c}\left\langle\Delta n_{\theta}\right\rangle \\
\times 10^{-4}\end{array}$ \\
\hline-3 & 611. & -4 & -3 & -3.3 & 1.9 & 1.5 & 8.1 & 6.3 \\
-4 & 63.2 & -5 & -4 & -3.3 & 1.4 & 1.0 & 7.8 & 6.0 \\
-5 & 7.06 & -6 & -5 & -3.3 & 0.95 & 0.48 & 6.8 & 4.9 \\
-6 & 1.13 & -6 & -5 & -3.7 & .47 & .049 & 0.023 & 0.22 \\
-7 & 0.446 & -6 & -5 & -3.7 & .025 & .019 & .085 & .082 \\
-8 & .331 & -6 & -5 & -3.7 & .0 & .013 & .063 & .060 \\
-9 & .303 & -6 & -5 & -3.7 & .0 & .013 & .058 & .055 \\
\hline
\end{tabular}

\section{TABLE 10C}

Optical path length change and stress birefringence for Pt inclusions in Glass(B). The notation of this table is the same as that for table 10a. The radius is $r_{0}=10^{-3} \mathrm{~cm}$.

\begin{tabular}{c|c|c|c|c|c|c|c|c}
\hline \hline $\begin{array}{c}\log _{10}(\tau / 3) \\
(\tau \text { in s })\end{array}$ & $\begin{array}{c}E_{l_{1}} \\
\left(\mathrm{~J} / \mathrm{cm}^{2}\right)\end{array}$ & $\begin{array}{c}\log _{10}\left(t_{1} / 3\right) \\
\left(t_{1} \text { in s }\right)\end{array}$ & $\begin{array}{c}\log _{10}\left(t_{2} / 3\right) \\
\left(t_{2} \text { in s }\right)\end{array}$ & $\begin{array}{c}\log _{10}\left(r_{2}\right) \\
\left(r_{2} \text { in cm }\right)\end{array}$ & $\begin{array}{c}\Delta L\left(r_{0}, r_{0}, \tau\right) \\
(\mathrm{cm}) \times 10^{-6}\end{array}$ & $\begin{array}{c}\langle\Delta L\rangle \\
(\mathrm{cm}) \times 10^{-6}\end{array}$ & $\begin{array}{c}\left\langle\Delta n_{r}\right\rangle \\
\times 10^{-4}\end{array}$ & $\begin{array}{c}\left\langle\Delta n_{\theta}\right\rangle \\
\times 10^{-4}\end{array}$ \\
\hline-3 & 70.6 & -4 & -3 & -2.52 & 9.5 & 6.3 & 9.5 & 7.1 \\
-4 & 11.3 & -4 & -3 & -2.52 & 4.7 & 0.78 & 6.9 & 3.9 \\
-5 & 4.46 & -4 & -3 & -2.52 & 0.25 & .68 & 1.8 & 1.5 \\
-6 & 3.31 & -4 & -3 & -2.52 & .0 & .52 & 1.3 & 1.1 \\
-7 & 3.03 & -4 & -3 & -2.52 & .0 & .48 & 1.2 & 9.7 \\
-8 & 2.95 & -4 & -3 & -2.52 & .0 & .47 & 1.2 & 9.4 \\
-9 & 2.93 & -4 & -4 & -3.52 & .0 & .46 & 1.2 & 9.4 \\
\hline
\end{tabular}

The data from tables 6,7 , and 10 also may be used to estimate the lens effect due to heated regions of the host. Whenever the refractive index increases with temperature $\left(d n_{h} / d T_{h}\right)>0$, the heated region of the host surrounding the inclusion might focus the same laser pulse or a succeeding laser pulse. Computing the effective focal length for a spherical shell with a spatially varying refractive index is too complicated for an estimate of the effect. Instead the following crude approximation is considered. The focal length $f$ of a spherical lens having a radius $r$ and an uniform refractive index $n_{1}$ is

$$
f=\left(n_{1} / n_{0}\right) r /\left[2\left\{\left(n_{1} / n_{0}\right)-1\right\}^{2}\right],
$$

where $n_{0}$ is the refractive index of the medium in which the spherical lens is imbedded. A measure of the expected importance of the spherical lens effect is the spatial extent of the deviation of the refractive index from its value before the laser pulse occurs, $n_{h}$. 
That is, the importance of the lens effect is measured in some manner by the quantity,

$$
\langle\delta n\rangle=\lim _{\rho \rightarrow \infty} \frac{1}{\rho} \int_{r_{0}}^{\rho}\left\{n_{h}(r, t)-n_{h}\right\} d r .
$$

A representative distance for the spatial extent is

$$
\langle r\rangle=\lim _{\rho \rightarrow \infty} \frac{1}{\rho} \int_{r_{0}}^{\rho} r\left\{n_{h}(r, t)-n_{h}\right\} d r,
$$

where the above integrals are finite. The quantity $\left[n_{r}(r, t)-n_{h}\right]$ is proportional to the temperature $T_{h}(r, t)$; namely, $\left[n_{h}(r, t)-n_{h}\right] \sim\left(d n_{h} / d T_{h}\right) T_{h}(r, t)$. When $\langle\delta n\rangle \simeq \leqslant n_{h}$, then an estimate for the focal length is

$$
f \sim n_{h}^{2}\langle r\rangle / 2\langle\delta n\rangle^{2}
$$

where

and

$$
\langle r\rangle=\left(d n_{h} / d T_{h}\right) \lim _{\rho \rightarrow \infty} \frac{1}{\rho} \int_{r_{0}}^{\rho} r T_{h}(r, t) d r
$$

$$
\langle\delta n\rangle=\left(d n_{h} / d T_{h}\right) \lim _{\rho \rightarrow \infty} \frac{1}{\rho} \int_{r_{0}}^{\rho} T_{h}(r, t) d r .
$$

Some typical numerical examples for platinum are as follows: when $r_{0}=1 \mu, E_{L}=20\left(\mathrm{~J} / \mathrm{cm}^{2}\right)$, and $\tau=30 \mathrm{~ns}$, then the maximum values for $\langle r\rangle$ and $\langle\delta n\rangle$ occur at times $t \sim 3 \mu \mathrm{s}$ and are respectively $\langle r\rangle \sim 2 \mu$ and $\langle\delta n\rangle \sim 4 \times 10^{-3}$. These values give an effective focal length of about $f \sim 14 \mathrm{~cm}$. But from tables 6 and 7 the tensile stress at $t=\tau=30 \mathrm{~ns}$ exceeds the theoretical strength of the host before the lens effect becomes most important. Consider now another example for platinum in which $\sigma_{\theta \theta}$ (max-tensile) is less than the theoretical breaking stress of the glass host. When $r_{0}=1 \mu, E_{L}=0.33\left(\mathrm{~J} / \mathrm{cm}^{2}\right), \tau=30 \mathrm{~ns}$, and $T_{h}\left(r_{0}, \tau\right)=600{ }^{\circ} \mathrm{C}$, then the maximum values of $\langle r\rangle$ and $\langle\delta n\rangle$ occur at times about $3 \mu$ s and are respectively $\langle r\rangle \sim 2 \mu$ and $\langle\delta n\rangle \sim 1.2 \times 10^{-4}$. These values give an effective focal length of about $f \sim 1.6 \times 10^{4} \mathrm{~cm}$. These focal lengths are very long.

Hence the model predicts that the lens effect arising from heated inclusions probably does not cause damage. Those cases for which the maximum tensile strength is less than the theoretical tensile stress of the glass have minimum effective focal lengths which are much greater than any dimension of neodymium doped glass elements used in present glass laser systems. In those cases for which the tensile stress exceeds the theoretical strength, the tensile stress probably causes damage before the lens effect could cause damage by heating another inclusion or by initiating an intrinsic damage mechanism such as self-focussing.

Equations (34) and (35) and the results in tables 6 and 7 show that $\mathrm{Sb}$ and the fictitious $\mathrm{Al}_{2} \mathrm{O}_{3}$ (a) are more likely to cause damage than $\mathrm{Pt}$. However, this difference is marginal. Additional computations for $\mathrm{Pt}$ spheres in fuzed silica, $\delta \alpha_{\text {eff }} \sim+6.0 \times 10^{-6}\left(1 /{ }^{\circ} \mathrm{C}\right)$; indicate that the maximum tensile stresses occur at $r=r_{0}$ and $t=\tau$ and that they are comparable to those stresses reported in tables 6 and 7 . However, the range of $r_{0}$ for which $\sigma_{\theta \theta}$ (max-tensile) exceeds the theoretical strength is much greater; namely, when

$$
10^{-4} \mathrm{~cm} \geqslant r_{0} \geqslant 10^{-6} \mathrm{~cm},
$$

$\tau=30 \mathrm{~ns}$, and $E_{L}=20\left(\mathrm{~J} / \mathrm{cm}^{2}\right)$, then $\sigma_{\theta \theta}$ (max-tensile; $\mathrm{Pt}$ in silica) $>6 \times 10^{9}\left(\mathrm{~N} / \mathrm{m}^{2}\right)$. Fused silica has a tensile strength comparable to glass.

The maximum tensile stress as a function of the thermal conductivity $K_{h}$ and the thermal expansion coefficient $\alpha_{h}$ is studied also. These investigations are limited to Pt in Glass (B) and in Glass (U) for which the effective expansion coefficients are negative. It is found that increasing $K_{h}$ in table 2 for Glass (B) from 0.008 to $0.04\left(\mathrm{~W} / \mathrm{cm}{ }^{\circ} \mathrm{C}\right)$ decreases the maximum tensile stress from $9.0 \times 10^{9}\left(\mathrm{~N} / \mathrm{m}^{2}\right)$ to $4.0 \times 10^{9}\left(\mathrm{~N} / \mathrm{m}^{2}\right)$. Similarly, increasing $K_{h}$ in table 2 for Glass (U) from 0.013 to $0.04\left(\mathrm{~W} / \mathrm{cm}{ }^{\circ} \mathrm{C}\right)$ decreases the maximum tensile stress from $10.6 \times 10^{9}\left(\mathrm{~N} / \mathrm{m}^{2}\right)$ to $5.8 \times 10^{9}\left(\mathrm{~N} / \mathrm{m}^{2}\right)$. Again, all the other properties of Glass (B) and of Glass (U) are kept the same except for the thermal expansion coefficient and the resulting maximum tensile stress as a function of $\alpha_{h}$ for fixed $r=5 \times 10^{-5} \mathrm{~cm}, \tau=30 \mathrm{~ns}$, and $E_{L}=20 \mathrm{~J} / \mathrm{cm}^{2}$ is reported in tables 8 and 9 . Observe that the maximum tensile stress has a minimum at $\alpha_{h}=\alpha_{h}(\min ) ;$ namely, $\alpha_{h}(\min$, Glass $(\mathrm{B})) \sim 10 \times 10^{-6}$ $\left(1 /{ }^{\circ} \mathrm{C}\right)$ and $\alpha_{h}(\min$, Glass $(\mathrm{U})) \sim 8 \times 10^{-6}\left(1 /{ }^{\circ} \mathrm{C}\right)$. Also, these tables indicate that the maximum tensile stress is a slowly varying function of the thermal expansion coefficient, $\alpha_{h}$. Thus, for these cases, $\alpha_{h}$ influences greatly the behavior near the interface because it appears in the effective expansion coefficient and the thermal conductivity influences greatly the behavior near the region of maximum tensile stress.

Intuitive arguments exist to explain why the temperature $T_{h}\left(r_{0}, t\right)$ in tables 6 and 7 has a maximum value at some $r_{0}=r\left(\max T_{h}\right)$ for fixed $E_{L}$ and $\tau$ and decreases for values of $r_{0}$ greater than $r\left(\max T_{h}\right)$ and less than $r\left(\max T_{h}\right)$. As $\left(r_{0} / r\left(\max T_{h}\right)\right)$ becomes larger than one, the volume increases much faster than the surface area of the sphere. The inclusion receives less energy per unit volume. Therefore, the maximum surface temperature at the end of the pulse decreases. As $\left(r_{0} / r\left(\max T_{h}\right)\right)$ becomes smaller than one, the equilibration time $\left(r_{0}^{2} / a_{h}^{2}\right)$ approaches zero. Then the surface temperature at the end of the pulse cannot deviate much from the equilibrium temperature due to the extremely short equilibration time.

The above computations in table 10 suggest that examining incipient absorbing centers in laser glasses either by methods which employ the interference of two light rays (which experience different optical path length changes due to the local variation of the refractive index near the inclusion) or by methods which employ thermal stress birefringence are promising. However, because the propagation of light in the presence of a stress birefrigence which has spherical symmetry is complex, the interpretation of birefringence data will be more tedious than data from an interference method. The feasibility of combining the inter- 
ference method and holographic techniques has been demonstrated for 50 micron particles [2]. In addition the results of table 10 suggest that the use of laser pulses with pulse widths greater than a few microseconds may be more promising for the detection of small incipient absorbing centers than the use of nanosecond laser pulses. The longer pulses produce spatial changes in the refractive index which extend over greater distances in the host and thereby increase the probability of detecting small inclusions. Another approach is to employ pulse widths and observation times which are less than the relaxation times of the host. Relaxation times for laser glasses between 800 ${ }^{\circ} \mathrm{C}$ and $1100{ }^{\circ} \mathrm{C}$ are approximately between nanoseconds and microseconds. These are estimates of the time during which stress is proportional to strain in glasses which are not elastic for infinite time. Such short time observations will permit one to raise the inclusion surface temperature substantially above the present $600{ }^{\circ} \mathrm{C}$ reported here and still satisfy the assumptions of the present model.

The author thanks especially Alan D. Franklin for many helpful discussions and for his encouragement. While undertaking this study the author also had several discussions with many other researchers concerned with laser damage problems, some of whom are cited in the references. He thanks them and the others from whom he has learned about damage in laser materials. He also thanks M. J. Cooper and A. Kahn for their reading of the manuscript.

\section{Appendix. Center-Host Interface}

A laser beam with an energy flux per unit area $I$ $\left(\mathrm{J} / \mathrm{cm}^{2} \mathrm{~s}\right)$, with a pulse width $\tau(\mathrm{s})$ and with a wavelength $\lambda_{L}(\mathrm{~cm})$ impinges upon the inclusion. The inclusion exhibits an absorptance $A(\lambda, T)$, a reflectance $R(\lambda, T)$, and a partial emissivity $E(\lambda, T)$, where $\lambda$ is the wavelength and $T$ is the absolute temperature of the inclusion. The following relations among $A, R$, and $E$ exist:

(a) $A+R=1$; no energy is transmitted.

(b) $E(\lambda, T) / A(\lambda, T)=e(\lambda, T)$; Kirchoff's law.

The function $e(\lambda, T)$ is a universal function only of $\lambda$ and $T$ and is independent of material and surface properties.

(c) $\int_{0}^{\infty} e(\lambda, T) d \lambda=S_{B} T^{4}$; Stefan-Boltzmann law. The constant $S_{B}$ is $S_{B}=5.673 \times 10^{-5}\left(\mathrm{erg} / \mathrm{cm}^{2} \mathrm{~s} \mathrm{~K}^{4}\right)$.

(d) $\lambda_{\max } T=0.2897 \mathrm{~cm} \mathrm{~K}$; Wien's displacement law. The wavelength for which $e(\lambda, T)$ has a maximum value for a fixed temperature $T$ is $\lambda_{\max }$.

The values for the absorptance $A(\lambda, T)$ in table 4 are valid for $r_{0} \gtrsim \lambda \sim 1.06 \mu$. The results of Mie scattering theory [11] show that $A\left(\lambda, T_{0}\right)$ does not change appreciably for values of $r_{0} \lesssim \lambda$ and $r_{0} \gtrsim \gamma^{-1}$ where $\gamma$ is the absorption coefficient.
The spherical inclusion has a complex index of refraction $m_{c}=n_{c}-i n_{c}^{\prime}$ and the Poynting vector for the incident radiation in the absorbing center is proportional to the factor exp $\left(-\gamma_{c}\left(r_{0}-r\right)\right)$. The absorption coefficient $\gamma_{c}$ is given by $\left(4 \pi n_{c}^{\prime} / \lambda_{L}\right)$. Because a sphere with index $m_{c}$ is imbedded in the host with a real index $m_{h}=n_{h} \approx 1.52$, the values for $m_{c}$ to use in the equations of ref. [11] are $m_{c}(\mathrm{med})=\left(m_{c} / n_{h}\right)$. Similarly the wavelength in the host is $\lambda_{L}$ (med) $=\left(\lambda_{I} / m_{h}\right)$ where $\lambda_{L}=1.06 \mu$. The sphere intercepts in accordance with Mie scattering theory $Q_{\mathrm{ext}} \pi r_{0}^{2} I$ watts from the incident laser beam, independently of the polarization of the beam. There will be $Q_{\mathrm{abs}} \pi r_{0}^{2} I$ watts absorbed by the sphere and $Q_{\text {sca }} \pi r_{0}^{2} I$ watts scattered in all directions by the sphere. The conservation of energy gives $Q_{\text {ext }}=Q_{\text {abs }}+Q_{\text {sca }}$. The absorptance $Q_{\text {abs }}=A(\lambda, T ;$ $r_{0}=$ small $)$ replaces the absorptance $A\left(\lambda, T ; r_{0}=\right.$ large $)$ quoted in table 4 , whenever $r_{0}$ becomes less than the wavelength $\lambda_{l}$ (med). The variable $X=\left(2 \pi r_{0} / \lambda_{L}(\right.$ med $\left.)\right)$ is introduced and when $X \lesssim 0.6$ Mie scattering theory relates $X$ and $Q_{\text {ext }}$; namely,

$$
Q_{\mathrm{ext}} \approx Q_{\mathrm{abs}} \approx 2.68 X \text { and } Q_{\mathrm{sca}} \ll Q_{\mathrm{abs}} .
$$

for platinum spheres in glass $\left(n_{h}=1.52\right)$ and

$$
Q_{\mathrm{ext}} \approx Q_{\mathrm{abs}} \approx 0.992 X
$$

for antimony spheres in glass $\left(n_{h}=1.52\right)$. When

then

$$
\lambda=1.06 \mu, X=9.01 \times 10^{4} \mathrm{~cm}^{-1} r_{0}
$$

and

$$
Q_{\text {abs }}(\mathrm{Pt}) \approx 24.1 \times 10^{4} \mathrm{~cm}^{-1} r_{0}
$$

$$
Q_{\text {abs }}(\mathrm{Sb}) \approx 8.93 \times 10^{4} \mathrm{~cm}^{-1} r_{0}
$$

for values of $r_{0} \lesssim 6.7 \times 10^{-6} \mathrm{~cm}$. Hence, $A\left(\lambda_{L}, T\right)$ does not change by more than a factor of three for values of $r_{0} \gtrsim \gamma_{c}^{-1}$.

\section{References}

[1] Bliss, E. S., in Damage in Laser Glass, A. J. Glass and A. H. Guenther, Eds. (ASTM Special Technical Publication 469, 1969), p. 9.

[2] Snitzer, E., private communication.

[3] Turnbull, D., private communication.

[4] Dietz, E. D., private communication.

[5] Mauer, R. D., private communication.

[6] Boley, B., and Weiner, J., Theory of Thermal Stresses (John Wiley Sons, Inc., New York, 1960).

[7] Bennett, H. S., Heat diffusion near absorbing centers in laser materials, J. Res. Nat. Bur. Stand. (U.S.), 75 A (Physics and Chem.), No. 4, 261 (July-Aug. 1971).

[8] Gilman, J. J., in Physics and Chemistry of Ceramics, C. Klingsberg, Ed. (Gordon and Breach, New York, 1963), p. 240.

[9] Quelle, F., J. Applied Optics 5, 633 (1966).

[10] Uhlmann, D., private communication.

[11] van de Hulst, H. C., Light Scattering by Small Particles (John Wiley and Sons, Inc., New York, 1957).

(Paper 75A4-666) 